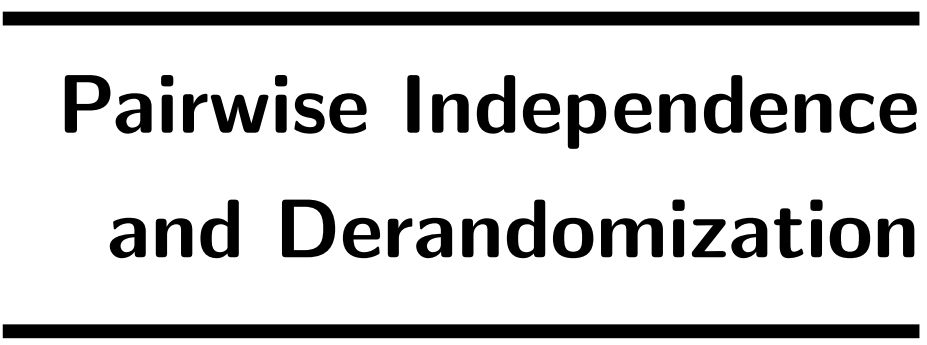





\title{
Pairwise Independence and Derandomization
}

\author{
Michael Luby \\ Digital Fountain \\ Fremont, CA, USA \\ Avi Wigderson \\ Institute for Advanced Study \\ Princeton, NJ, USA \\ avi@ias.edu
}

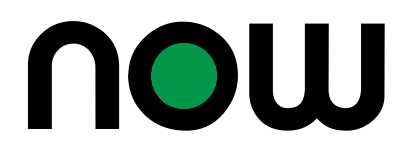

the essence of knowledge

Boston - Delft 


\title{
Foundations and Trends ${ }^{\circledR}$ in Theoretical Computer Science
}

\author{
Published, sold and distributed by: \\ now Publishers Inc. \\ PO Box 1024 \\ Hanover, MA 02339 \\ USA \\ Tel. +1-781-985-4510 \\ www.nowpublishers.com \\ sales@nowpublishers.com \\ Outside North America: \\ now Publishers Inc. \\ PO Box 179 \\ 2600 AD Delft \\ The Netherlands \\ Tel. +31-6-51115274
}

A Cataloging-in-Publication record is available from the Library of Congress

The preferred citation for this publication is M. Luby and A. Wigderson, Pairwise Independence and Derandomization, Foundation and Trends ${ }^{\circledR}$ in Theoretical Computer Science, vol 1, no 4, pp 237-301, 2005

Printed on acid-free paper

ISBN: 1-933019-22-0

(C) 2006 M. Luby and A. Wigderson

All rights reserved. No part of this publication may be reproduced, stored in a retrieval system, or transmitted in any form or by any means, mechanical, photocopying, recording or otherwise, without prior written permission of the publishers.

Photocopying. In the USA: This journal is registered at the Copyright Clearance Center, Inc., 222 Rosewood Drive, Danvers, MA 01923. Authorization to photocopy items for internal or personal use, or the internal or personal use of specific clients, is granted by now Publishers Inc for users registered with the Copyright Clearance Center (CCC). The 'services' for users can be found on the internet at: www.copyright.com

For those organizations that have been granted a photocopy license, a separate system of payment has been arranged. Authorization does not extend to other kinds of copying, such as that for general distribution, for advertising or promotional purposes, for creating new collective works, or for resale. In the rest of the world: Permission to photocopy must be obtained from the copyright owner. Please apply to now Publishers Inc., PO Box 1024, Hanover, MA 02339, USA; Tel. +1 781871 0245; www.nowpublishers.com; sales@nowpublishers.com

now Publishers Inc. has an exclusive license to publish this material worldwide. Permission to use this content must be obtained from the copyright license holder. Please apply to now Publishers, PO Box 179, 2600 AD Delft, The Netherlands, www.nowpublishers.com; e-mail: sales@nowpublishers.com 


\title{
Foundations and Trends ${ }^{\circledR}$ in Theoretical Computer Science
}

Volume 1 Issue 4, 2005

\section{Editorial Board}

\author{
Editor-in-Chief: \\ Madhu Sudan \\ Department of $C S$ and $E E$ \\ MIT, Stata Center, Room G640 \\ 32 Vassar Street, Cambridge \\ Massachusetts 02139, \\ USA \\ madhu@mit.edu

\section{Editors} \\ Bernard Chazelle (Princeton) \\ Oded Goldreich (Weizmann Inst.) \\ Shafi Goldwasser (MIT and Weizmann Inst.) \\ Jon Kleinberg (Cornell University) \\ László Lovász (Microsoft Research) \\ Christos Papadimitriou (UC. Berkeley) \\ Prabhakar Raghavan (Verity Inc.) \\ Peter Shor (MIT) \\ Madhu Sudan (MIT) \\ Éva Tardos (Cornell University) \\ Avi Wigderson (IAS)
}




\section{Editorial Scope}

\section{Foundations and Trends ${ }^{\circledR}$ in Theoretical Computer Science}

will publish survey and tutorial articles in the following topics:

- Algorithmic game theory

- Computational algebra

- Computational aspects of combinatorics and graph theory

- Computational aspects of communication

- Computational biology

- Computational complexity

- Computational geometry

- Computational learning

- Computational Models and Complexity
- Computational Number Theory

- Cryptography and information security

- Data structures

- Database theory

- Design and analysis of algorithms

- Distributed computing

- Information retrieval

- Operations Research

- Parallel algorithms

- Quantum Computation

- Randomness in Computation

\section{Information for Librarians}

Foundations and Trends ${ }^{\circledR}$ in Theoretical Computer Science, 2005, Volume 1, 4 issues. ISSN paper version 1551-305X. ISSN online version 1551-3068. Also available as a combined paper and online subscription. 
Foundations and Trends ${ }^{\circledR}$ in

Theoretical Computer Science

Vol. 1, No 4 (2005) 237-301

(C) 2006 M. Luby and A. Wigderson

DOI: $10.1561 / 0400000009$

\title{
Pairwise Independence and Derandomization
}

\author{
Michael Luby ${ }^{1}$ and Avi Wigderson ${ }^{2}$ \\ 1 Digital Fountain, Fremont, CA, USA \\ 2 Institute for Advanced Study, Princeton, NJ, USA, avi@ias.edu
}

\begin{abstract}
This article gives several applications of the following paradigm, which has proven extremely powerful in algorithm design and computational complexity. First, design a probabilistic algorithm for a given problem. Then, show that the correctness analysis of the algorithm remains valid even when the random strings used by the algorithm do not come from the uniform distribution, but rather from a small sample space, appropriately chosen. In some cases this can be proven directly (giving "unconditional derandomization"), and in others it uses computational assumptions, like the existence of 1-way functions (giving "conditional derandomization").

The article is based on a series of lectures given by the authors in 1995, where the notes were scribed by the attending students. (The detailed list of scribes and other contributors can be found in the Acknowledgements section at the end of the manuscript.) The current version is essentially the same, with a few minor changes. We note that this publication takes place a decade after the lectures were given. Much has happened in the area of pseudorandomness and derandomization since, and perhaps a somewhat different viewpoint, different material, and different style would be chosen were these lectures given today. Still, the material presented is self contained, and is a prime
\end{abstract}


manifestation of the "derandomization" paradigm. The material does lack references to newer work though. We recommend the reader interested in randomness, derandomization and their interplay with computational complexity to consult the following books and surveys, as well as their extensive bibliography: [31, 14, 36, 37, 21, 42]. 


\section{Contents}

1 Pairwise Independence 1

1.1 Pairwise independence: Definition 2

1.2 Small families of hash functions 3

1.3 Derandomization applications 4

1.4 Dictionaries 5

2 Limited Independence Probability Spaces 9

2.1 Modulo prime space 9

$\begin{array}{lll}2.2 & \text { Linear polynomial space } & 10\end{array}$

2.3 Mapping between $\{0,1\}^{n}$ and GF $\left[2^{n}\right] \quad 11$

2.4 Inner product space 11

3 Pairwise Independence and Complexity Classes 13

3.1 RP and BPP 13

$\begin{array}{lll}3.2 & \text { Complexity of unique solutions } & 15\end{array}$

$3.3 \mathrm{BPP} \subseteq \sum_{2} \quad 16$

$3.4 \mathrm{AM}=\mathrm{IP} \quad 18$

4 Recycling Randomness $\quad 21$

4.1 Deterministic amplification 21 
4.2 The Chor-Goldreich generator 23

4.3 The Nisan generator 24

4.4 The Impagliazzo-Zuckerman generator 26

4.5 The expander mixing Lemma 29

4.6 The Karp-Pippenger-Sisper generator 32

4.7 The Ajtai-Komlós-Szemerédi generator 32

5 Pseudo-Random Generators $\quad 35$

5.1 One-way functions 35

5.2 Hidden Bit Theorem 38

5.3 Pseudo-random generators 44

6 Deterministic Counting $\quad 47$

6.1 \#P and approximate counting 47

6.2 DNF counting 50

$\begin{array}{ll}\text { 6.3 GF[2] polynomial counting } & 51\end{array}$

6.4 Bounded depth circuit counting 55

$\begin{array}{ll}\text { Acknowledgements } & 63\end{array}$

$\begin{array}{ll}\text { References } & 65\end{array}$ 
In this chapter, and indeed in much of the rest of this article, we will be considering randomized algorithms, and their performance when their input is not "purely" random. To set the stage, let us consider an algorithm $A$ that on input $x$ wishes to evaluate some function $f$ at $x$. A randomized algorithm for this task may use an input a sequence of random variables $Z_{1}, \ldots, Z_{n}$ where the $Z_{i}$ 's take their value from some finite set $T$. Informally, $A$ would be considered good for computing $f$, if it is very likely to output $f(x)$, when the $Z_{i}$ 's are drawn independently and uniformly from the set $T$. But what happens, when the random variables are not chosen uniformly, and especially, independently. Depending on the level of independence, the support of the joint distribution on $Z_{1}, \ldots, Z_{n}$ could be much smaller than the support of the independent uniform distribution. In turn this allows for efficient calculation of the probability of various events (or to compute the most likely output of $A$ on input $x$ ). In this section, we introduce definitions that study distributions that are not fully independent, and show some algorithmic uses. 
2 Pairwise Independence

\subsection{Pairwise independence: Definition}

Consider a set of $N$ random variables indexed by a set $U$, i.e., $\left\{Z_{x}\right.$ : $x \in U\}$ with $|U|=N$, that take on values from a set $T$, (i.e., $Z_{x} \in T$ ). Let $D: T^{U} \rightarrow[0,1]$ denote their joint distribution function, i.e., for $\alpha=$ $\left\{\alpha_{x} \in T \mid x \in U\right\}$ let $\operatorname{Pr}\left[\forall x \in U, Z_{x}=\alpha_{x}\right]=D(\alpha)$.

For finite $t=|T|$, a uniform distribution (i.e., $D(\alpha)=1 / t^{n}$ ) assigns $\operatorname{Pr}\left[Z_{x}=\alpha_{x}\right]=1 / t$, for all $x \in U, \alpha_{x} \in T$. If this distribution satisfies, for all $x \neq y \in U$ and for all $\alpha, \beta \in T$,

$$
\operatorname{Pr}\left[Z_{x}=\alpha, Z_{y}=\beta\right]=\operatorname{Pr}\left[Z_{x}=\alpha\right] \cdot \operatorname{Pr}\left[Z_{y}=\beta\right]=1 / t^{2},
$$

then we refer to this distribution as pairwise independent.

Pairwise independence is not the same as complete independence. For example, consider following set of three pairwise-independent binary variables $(U=\{1,2,3\}, T=\{0,1\}, t=2)$, where each row gives an assignment to the three variables and the associated probability. (The leftmost column gives an index $s \in\{0,1\}^{2}$ for each row.)

\begin{tabular}{c|cccc}
$s$ & $Z_{1}$ & $Z_{2}$ & $Z_{3}$ & $D(\cdot)$ \\
\hline 00 & 0 & 0 & 0 & $\frac{1}{4}$ \\
01 & 0 & 1 & 1 & $\frac{1}{4}$ \\
10 & 1 & 0 & 1 & $\frac{1}{4}$ \\
11 & 1 & 1 & 0 & $\frac{1}{4}$
\end{tabular}

The above distribution has its support on only four elements of $T^{U}$, whereas the uniform distribution has support on all eight elements. (The support of a distribution $D()$ is the set of elements $\alpha \in T^{U}$ for which $D(\alpha)>0$.) Shortly, we will see that the support of pairwise independent distrubtions can get much smaller than the support of the uniform distribution, as $N=|U| \rightarrow \infty$.

The notion of pairwise independence emerged first in the context of "hash functions". To describe this context, notice that each row $s$ above can be thought of as a function $h_{s}: U \rightarrow T$, where $h_{s}(x)=Z_{x}$. Let $\mathcal{S}$ be the set of indices $s$ for these functions. So, in this case, $\mathcal{S}=\{0,1\}^{2}$. For all $x \neq y \in U$, for all $\alpha, \beta \in T$, we have

$$
\operatorname{Pr}_{s \in R_{\mathcal{S}}}\left[h_{s}(x)=\alpha \wedge h_{s}(y)=\beta\right]=1 / 4=1 / t^{2}
$$


(where the notation $s \in_{R} \mathcal{S}$ denotes that $s$ is chosen uniformly at random from the set $\mathcal{S}$ ). (Notice in particular that $\operatorname{Pr}_{s \in R_{R}}\left[h_{s}(x)=h_{s}(y)\right]=$ $1 / 2=1 / t$.) Any set of functions satisfying this condition is a 2-universal family of hash functions. Definitions and explicit constructions of 2universal hash functions were first given by Carter-Wegman [40]. The original applications described in Carter-Wegman [40] were straightforward, similar to those described in the later section on dictionaries based on hashing. As these notes indicate, subsequently 2-universal hashing has been applied in surprising ways to a rich variety of problems.

\subsection{Small families of hash functions}

In the last section we saw how to construct $N=3$ pairwise independent random variables with a smaller support than needed for 3 fully independent random variables. (Of course, we couldn't have picked a smaller choice of $N$ to demonstrate this difference!) But to get a true sense of the difference, it is useful to let $N \rightarrow \infty$. In this section we will show how to construct $N=2^{n}$ random variables, indexed by the set $U=\{0,1\}^{n}$ taking on values in the set $T$ which is also chosen to be $\{0,1\}^{n}$.

One simple way to construct a family of hash functions mapping $\{0,1\}^{n} \rightarrow\{0,1\}^{n}$ is to let $\mathcal{S}=\{0,1\}^{n} \times\{0,1\}^{n}$, and then for all $s=$ $(a, b) \in \mathcal{S}$, for all $x \in\{0,1\}^{n}$ define $h_{s}(x)=a x+b$, where the arithmetic operations are with respect to the finite field GF $\left[2^{n}\right]$. Thus, each $h_{s}$ maps $\{0,1\}^{n} \rightarrow\{0,1\}^{n}$ and $\mathcal{S}$ is the index set of the hash functions. For each $s=(a, b) \in \mathcal{S}$, we can write:

$$
\left(\begin{array}{l}
h_{s}(x) \\
h_{s}(y)
\end{array}\right)=\left(\begin{array}{ll}
x & 1 \\
y & 1
\end{array}\right)\left(\begin{array}{l}
a \\
b
\end{array}\right)
$$

When $x \neq y$, the matrix is non-singular, so that for any $x, y \in\{0,1\}^{n}$, the pair $\left(h_{s}(x), h_{s}(y)\right)$ takes on all $2^{2 n}$ possible values (as $s$ varies over all $\mathcal{S}$ ). Thus if $s$ is chosen uniformly at random from $\mathcal{S}$, then $\left(h_{s}(x), h_{s}(y)\right)$ is also uniformly distributed. This property of hash functions is called 2-universal. 
We can view $\mathcal{S}$ as the set of points in a sample space on the set of random variables $\left\{Z_{x}: x \in\{0,1\}^{n}\right\}$ where $Z_{x}(s)=h_{s}(x)$ for all $s \in S$. With respect to the uniform distribution on $S$, these random variables are pairwise independent, i.e., for all $x \neq y \in\{0,1\}^{n}$, for all $\alpha, \beta \in\{0,1\}^{n}$

$$
\begin{aligned}
\operatorname{Pr}_{s \in R^{\mathcal{S}}}\left[Z_{x}(s)\right. & \left.=\alpha \wedge Z_{y}(s)=\beta\right] \\
& =\operatorname{Pr}_{s \in \in_{R} \mathcal{S}}\left[Z_{x}(s)=\alpha\right] \cdot \operatorname{Pr}_{s \in R_{R}^{\mathcal{S}}}\left[Z_{y}(s)=\beta\right]=1 / 2^{2 n} .
\end{aligned}
$$

To obtain a hash function that maps to $k<n$ bits, we can still use $\mathcal{S}$ as the function index family: The value of the hash function indexed by $s$ on input $x$ is obtained by computing $h_{s}(x)$ and using the first $k$ bits.

The imporant properties of these hash functions are:

- Pairwise independence.

- Succinctness - each function can be described as a $2 n$-bit string. Therefore, randomly picking a function index requires only $2 n$ random bits.

- The function $h_{s}(x)$ can easily be computed (in LOGSPACE, for instance) given the function index $s$ and the input $x$.

In the sequel, unless otherwise specified we are referring to this set of pairwise independent hash functions and $\mathcal{S}$ denotes the set of indices for the hash family.

\subsection{Derandomization applications}

Consider, for example, the MAXCUT problem: given a graph $G=$ $(V, E)$, find a two-coloring of the vertices $\chi: V \rightarrow\{0,1\}$ so as to maximize $c(\chi)=|\{(x, y) \in E: \chi(x) \neq \chi(y)\}|$. We describe a solution to this problem that is guaranteed to produce a cut where at least half the edges cross the cut.

If the vertices are colored randomly ( 0 or 1 with probability $1 / 2$ ) by choosing $\chi$ uniformly from the set of all possible $2^{|V|}$ colorings, then:

$$
E[c(\chi)]=\sum_{(x, y) \in E} \operatorname{Pr}[\chi(x) \neq \chi(y)]=\frac{|E|}{2}
$$


Thus, there must always be a cut of size at least $\frac{|E|}{2}$. Let $\mathcal{S}$ be the index set for the hash family mapping $V \rightarrow\{0,1\}$. Since the summation above only requires the coloring of vertices to be pairwise-independent, it follows that $E\left[c\left(h_{s}\right)\right]=\frac{|E|}{2}$ when $s \in_{R} \mathcal{S}$. Since $|\mathcal{S}|=|V|^{2}$, we can deterministically try $h_{s}$ for all $s \in \mathcal{S}$ in polynomial time (even in the parallel complexity class $\mathbf{N C}$ ), and for at least one $s \in \mathcal{S}, h_{s}$ defines a

partition of the nodes where at least $\frac{|E|}{2}$ edges cross the partition.

This derandomization approach was developed and discussed in general terms in the series of papers Chor-Goldreich [10], Luby [28], Alon-Babai-Itai [5]. There, the approach was applied to derandomize algorithms such as witness sampling, a fast parallel algorithm for finding a maximal independent set, and other graph algorithms.

\subsection{Dictionaries}

One application that uses hashing is in building "dictionaries". A dictionary is a data structure that represents a subset $N$ of size $|N|=n$ from some universe of possible words $U$ so as to be able support queries of the form " $x \in N$ ?", for $x \in U$. (This is a natural abstraction of the classical notion of a "dictionary" for, say, English where $U$ may be thought of as all sequences of upto 20 English letters, while $N$ is the subset which are actually words in English.) Deterministic schemes based on balanced tree data structures build such data structures in time $\mathcal{O}(n \log n)$ and subsequent look-ups in time $\mathcal{O}(\log n)$ each.

Random hashing can speed this up considerably. The simple use of hashing would be as follows. Let $T=\{1, \ldots, t\}$ be a set of sufficiently large cardinality, and let $h$ be some "hash" function mapping $U \rightarrow T$. We will store the elements of $N$ in an array $D[1, \ldots, t]$, with elements being indexed by elements of $T$. Our intent would be to set $D[h(x)]=x$ for every $x \in N$. Of course this would not be possible if we had "collisions", i.e., if $h(x)=h(y)$ for $x \neq y \in N$. Assuming $h$ is collision-free on $N$, easy to describe, that $h(x)$ can be computed in "constant" time, and assuming further that $D[1, \ldots, t]$ is initialized properly when we start, insertions and lookups take constant time. It turns out that by picking the hash function $h$ randomly from a small set (as described in Section 1.2) can now be used to remove most of 
these assumptions. In particular if we choose $s \in_{R} \mathcal{S}$, and use $h=h_{s}$ then the expected number of colliding pairs $\mathrm{C}$ may be bounded from above as follows:

$$
E[C]=\sum_{x \neq y \in N} \operatorname{Pr}_{s \in R}\left[h_{s}(x)=h_{s}(y)\right]=\left(\begin{array}{l}
n \\
2
\end{array}\right) \cdot \frac{1}{t}
$$

For instance, if $t=n^{2}$, then $E[C] \leq \frac{1}{2}$ (and so the probability that $h$ is 1 -to- 1 is $\geq \frac{1}{2}$ ). (If $t=n$, then $E[C] \leq \frac{n}{2}$.) Thus this yields a simple hashing scheme in which insertions and look-ups cost a unit time, though the size of the data structure representing $N$ is of size $O\left(n^{2}\right)$ (assuming that the table $D$ of size $n^{2}$ is initialized properly). Below we see how to improve upon the space complexity.

The following two-level hashing scheme, due to Fredman-KomlósSzemerédi [12], also takes time $\mathcal{O}(n)$ to construct the dictionary and constant time for each look-up, but the advantage is that it uses only $\mathcal{O}(n)$ cells in total. Let $T=\{1, \ldots, n\}$.

(1) Pick $s \in_{R} \mathcal{S}$ and map $N$ into $T$. For each $i \in T$, let $N_{i}$ be the subset of $N$ mapped to $i$ by $h_{s}$, and let $n_{i}=\left|N_{i}\right|$. Let $C=\sum_{i \in T}\left(\begin{array}{c}n_{i} \\ 2\end{array}\right)$ be the number of colliding pairs. If $C>n$ then start over at step (1), else go on to step (2).

(2) For each $i \in T$, if $n_{i} \geq 1$ then we allocate a table $T_{i}$ of $n_{i}^{2}$ cells, and let $\mathcal{S}_{i}$ denote the index set for the pairwise independent hash family (as in Section 1.2) mapping $U \rightarrow T_{i}$. Pick $s_{i} \in_{R} \mathcal{S}_{i}$, and use $h_{s_{i}}$ to map $N_{i}$ to $T_{i}$. If $h_{s_{i}}$ maps $N_{i} 1$ to- 1 into $T_{i}$ then this is a good choice for $s_{i}$, else rechoose $s_{i}$ independently and try again until $h_{s_{i}}$ does describe a 1-to-1 mapping.

Because $E[C] \leq n / 2$ in step (1), $\operatorname{Pr}[C \leq n] \geq 1 / 2$, and thus the expected number of times step (1) is repeated is at most 2. Similarly, in step (2), for each $i \in T$, the expected number of times till the mapping of $N_{i}$ into $T_{i}$ is 1-to-1 is at most 2 . Thus, the overall expected time to construct the dictionary is $\mathcal{O}(n)$. The total number of cells used to store $N$ is $D=\sum_{i \in T} n_{i}^{2}$. Noting that $D-2 C=|N|=n$, and that $C \leq n$, it 
follows that at most $3 n$ cells are used to store $N$. Note that we need to also store $s$ and all $s_{i}$ for all $i \in\{1, \ldots, n\}$, but this takes at most $2(n+1)$ additional cells, since the description of each hash function takes two cells.

Each find operation takes constant time. 

We describe constructions of probability spaces that induce limited independence on a sequence of random variables. These are extensions of constructions described in Section 1.2.

\subsection{Modulo prime space}

Let $p$ be a prime number. The sample space is the set of all pairs $\mathcal{S}=\left\{(a, b): a, b \in \mathcal{Z}_{p}\right\}$, where $\mathcal{Z}_{p}=\{0, \ldots, p-1\}$. The distribution on the sample points is uniform, i.e., $(a, b) \in_{R} \mathcal{S}$. Let $\zeta$ be an indeterminate and consider the polynomial

$$
p_{a, b}(\zeta)=(a \zeta+b) \bmod p
$$

where $(a, b) \in \mathcal{S}$. For all $i \in \mathcal{Z}_{p}$, define random variable

$$
X_{i}(a, b)=p_{a, b}(i)
$$

For brevity, we sometimes use $X_{i}$ in place of $X_{i}(a, b)$.

Claim 2.1. $X_{0}, \ldots, X_{p-1}$ are uniformly distributed in $\mathcal{Z}_{p}$ and pairwise independent. 
Proof. For any pair $i, j \in \mathcal{Z}_{p}, i \neq j$, and for any pair of values $\alpha, \beta \in \mathcal{Z}_{p}$, there is a unique solution $a, b \in \mathcal{Z}_{p}$ to the pair of equations:

- $p_{a, b}(i)=\alpha$.

- $p_{a, b}(j)=\beta$.

Thus, $\operatorname{Pr}_{A, B}\left[X_{i}(A, B)=\alpha \wedge X_{j}(A, B)=\beta\right]=1 / p^{2}$.

Recall the definition in Section 1.1 of pairwise independence. The following is a generalization of this definition.

Definition 2.2. ( $k$-wise independence) Let $X_{1}, \ldots, X_{m}$ be a sequence of random variables with values in a set $N$. We say the random variables are $k$-wise independent if, for all $1 \leq i_{1}<\cdots<i_{k} \leq m$ and for all $\alpha_{1}, \ldots, \alpha_{k} \in N$,

$$
\operatorname{Pr}\left[X_{i_{1}}=\alpha_{1} \wedge \cdots \wedge X_{i_{k}}=\alpha_{k}\right]=\operatorname{Pr}\left[X_{i_{1}}=\alpha_{1}\right] \cdots \operatorname{Pr}\left[X_{i_{k}}=\alpha_{k}\right] .
$$

Exercise 2.3. Let $p$ be a prime number and let $m \leq p$. Generalize the Modulo Prime Space to a probability space where $X_{0}, \ldots, X_{m-1} \in R$ are $k$-wise independent, where the size of the probability space is $p^{k}$.

The Modulo Prime Space can be generalized as follows. The following construction is a more detailed description of the one presented in Section 1.2.

\subsection{Linear polynomial space}

Let $\mathcal{F}$ be any finite field and consider the polynomial

$$
p_{a, b}(\zeta)=a \zeta+b
$$

over $\mathcal{F}$, where $a, b \in \mathcal{F}$ (we identify the integers $\{0, \ldots,|\mathcal{F}|-1\}$ with the elements of $\mathcal{F})$. The sample space is $\mathcal{S}=\{(a, b): a, b \in \mathcal{F}\}$ and the distribution on $\mathcal{S}$ is $(a, b) \in_{R} \mathcal{S}$. For all $i \in \mathcal{F}$, define random variable

$$
X_{i}(a, b)=p_{a, b}(i),
$$


where $i$ on the left side of the equality is treated as an index and on the right side of the equality it is the corresponding element of $\mathcal{F}$.

The random variables $X_{0}, \ldots, X_{|\mathcal{F}|-1}$ are uniformly distributed in $\mathcal{F}$ and pairwise independent. A field with nice properties is $\mathrm{GF}\left[2^{n}\right]$, the Galois field with $2^{n}$ elements.

\subsection{Mapping between $\{0,1\}^{n}$ and $\mathrm{GF}\left[2^{n}\right]$}

There is a natural mapping between $\{0,1\}^{n}$ and polynomials in one variable $\zeta$ of degree $n-1$ over GF[2]. Namely, if $a \in\{0,1\}^{n}$ and $\left\langle a_{0}, \ldots, a_{n-1}\right\rangle$ are the bits of $a$ then the corresponding polynomial is

$$
a(\zeta)=\sum_{i=0}^{n-1} a_{i} \zeta^{i}
$$

These polynomials are the field elements of $\mathrm{GF}\left[2^{n}\right]$. Let $a \in\{0,1\}^{n}$ and $b \in\{0,1\}^{n}$ and let $a(\zeta)$ and $b(\zeta)$ be the corresponding polynomials. Computing $a+b$ over $\mathrm{GF}\left[2^{n}\right]$ consists of computing $a \oplus b$, where $\oplus$ is vector addition over GF[2]. Equivalently, computing $a+b$ over GF $\left[2^{n}\right]$ consists of computing $a(\zeta)+b(\zeta)$ over GF[2], i.e., for all $i \in\{0, \ldots, n-1\}$, the $i$ th coefficient of $a(\zeta)+b(\zeta)$ is $a_{i} \oplus b_{i}$. Computing $a \cdot b$ over $\mathrm{GF}\left[2^{n}\right]$ consists of computing $a(\zeta) \cdot b(\zeta) \bmod r(\zeta)$, where $a(\zeta) \cdot b(\zeta)$ is polynomial multiplication over $\mathrm{GF}[2]$ that results in a polynomial of degree $2 n-2$, and $r(\zeta)$ is a fixed irreducible polynomial of degree $n$. The zero element of GF $\left[2^{n}\right]$ is the identically zero polynomial with coefficients $a_{i}=0$ for all $i \in\{0, \ldots, n-1\}$, and the identity element is the polynomial with coefficients $a_{0}=1$ and $a_{i}=0$ for all $i \in\{1, \ldots, n-1\}$.

In the Modulo Prime Space, $X_{0}, \ldots, X_{p-1}$ are pairwise independent and the size of the space is $p^{2}$. We describe a way to construct a pairwise independent probability space for $\{0,1\}$-valued random variables that has size linear in the number of random variables.

\subsection{Inner product space}

Let $\ell$ be a positive integer. The sample space is $\mathcal{S}=\{0,1\}^{\ell}$ and the distribution on sample points is $a \in_{R} \mathcal{S}$. For all $i \in\{0,1\}^{\ell} \backslash\left\{0^{\ell}\right\}$, define 
random variable

$$
X_{i}(a)=a \odot i=\left(\sum_{j=1}^{i} a_{j} \cdot i_{j}\right) \bmod 2 .
$$

(We use $\odot$ to denote multiplication of matrices over GF[2], where we are using the convention that a vector to the left of $\odot$ is considered a row vector and a vector to the right of $\odot$ is viewed as a column vector.)

Claim 2.4. $X_{1}, \ldots, X_{2^{\ell}-1}$ are uniformly distributed and pairwise independent.

Exercise 2.5. Prove the pairwise independence property for the Inner Product Space.

Exercise 2.6. Let $p$ be a positive integer and let $X_{1}, \ldots, X_{n} \in_{R} \mathcal{Z}_{p}$ be a sequence of four-wise independent random variables. Define random variable

$$
Y=\min \left\{\left(X_{i}-X_{j}\right) \bmod p: 1 \leq i<j \leq n\right\} .
$$

Prove there is a constant $c>0$ such that for any $\alpha \leq 1$

$$
\operatorname{Pr}\left[Y \leq \alpha p / n^{2}\right] \geq c \alpha .
$$

Hint 2.7. Let $N$ be the set of $n(n-1) / 2$ unordered pairs $\{(i, j): 1 \leq$ $i<j \leq n\}$. For fixed $\alpha$, consider the sequence of $\{0,1\}$-valued random variables $\left\{Z_{e}: e \in N\right\}$, where if $e=(i, j)$ then $Z_{e}=1$ if $\left|X_{i}-X_{j}\right| \leq$ $\alpha p / n^{2}$ and $Z_{e}=0$ otherwise. Using the first two terms of the inclusionexclusion formula, show that for any $\alpha$,

$\operatorname{Pr}\left[\exists e \in N: Z_{e}=1\right] \geq \sum_{e \in N} \operatorname{Pr}\left[Z_{e}=1\right]-\sum_{e, e^{\prime} \in N, e \neq e^{\prime}} \operatorname{Pr}\left[Z_{e}=1 \wedge Z_{e^{\prime}}=1\right]$. 


\section{Pairwise Independence and Complexity Classes}

The modern theory of computational complexity revolves around the notions of proofs, randomness and interaction. This leads to a rich collection of complexity classes (P, NP, $\Sigma_{i}^{P}, \Pi_{i}^{P}$, RP, BPP, AM, IP etc.). The formal definitions of the classes often suggests some obvious relationships between them such as $\mathcal{C}_{1} \subseteq \mathcal{C}_{2}$ for some pairs of classes $\mathcal{C}_{1}$ and $\mathcal{C}_{2}$, while leaving most other such relationships open. However, many non-obvious relationships turn out to hold between these classes, and many such are shown using the technique of pairwise independence. In this chapter we describe several such relationships.

This chapter, while technically self-contained, assumes the reader has some background in computational complexity (mostly for the motivation behind the classes). A reader is encouraged to read some classical texts in computational complexity $[34,37]$ before carrying on with this chapter.

\subsection{RP and BPP}

Recall that a language $\mathcal{L} \in \mathbf{N P}$ if there is a polynomial time TM $M$ (where TM denotes a Turing machine) with the following properties. 


\section{Pairwise Independence and Complexity Classes}

$M$ has two inputs $x$ and $y$, where $x$ is the string for which membership in $\mathcal{L}$ is trying to be decided, and $y$ is a potential witness for membership of $x$ in $\mathcal{L}$. If $x \in\{0,1\}^{n}$ then $y \in\{0,1\}^{r}$, where $r$ is polynomial in $n$. The output of $M(x, y)$ is a single bit. The running time of $M(x, y)$ is polynomial in $\|x\|$. For $x \in\{0,1\}^{n}$, let $W_{x}=\left\{y \in\{0,1\}^{r}: M(x, y)=\right.$ $1\}$. The machine has the property that for all $x \in\{0,1\}^{n}$,

$$
\begin{aligned}
& x \in \mathcal{L} \Rightarrow\left|W_{x}\right|>0, \\
& x \notin \mathcal{L} \Rightarrow\left|W_{x}\right|=0 .
\end{aligned}
$$

$\mathbf{R P}$ is the class of languages $\mathcal{L}$ where membership can be checked with one-sided error by a randomized, polynomial-time TM. Keeping the same notation as above, $\mathcal{L} \in \mathbf{R P}$ if there is TM $M$ with the following properties. There is a constant $\mathrm{c}_{\text {yes }}>0$ associated with $M$. For $x \in$ $\{0,1\}^{n}$, let $W_{x}=\left\{y \in\{0,1\}^{r}: M(x, y)=1\right\}$. For any $A \subseteq\{0,1\}^{r}$, let $\mu(A)=|A| / 2^{r}$ be the fraction of $r$-bit strings which are in $A$. $M$ has the property that for all $x \in\{0,1\}^{n}$,

$$
\begin{aligned}
& x \in \mathcal{L} \Rightarrow \mu\left(W_{x}\right) \geq c_{\mathrm{yes}}, \\
& x \notin \mathcal{L} \Rightarrow \mu\left(W_{x}\right)=0 .
\end{aligned}
$$

The way we can decide membership of $x \in\{0,1\}^{n}$ is to choose $y \in_{R}$ $\{0,1\}^{r}$ and decide $x \in \mathcal{L}$ if $M(x, y)=1$, i.e., if $y \in W_{x}$, and decide $x \notin \mathcal{L}$ if $M(x, y)=0$, i.e., if $y \notin W_{x}$. Notice that the decision is always correct if $x \notin \mathcal{L}$, but the decision is only correct with probability $c_{\text {yes }}$ if $x \in \mathcal{L}$. On the other hand, when $x \in \mathcal{L}$, if $y \in W_{x}$ then $y$ is a witness to the fact that $x$ really is in $\mathcal{L}$, i.e., we can have full confidence in our decision. The standard way to boost confidence in the decision is to choose $y_{1}, \ldots, y_{k} \in_{R}\{0,1\}^{r}$ and decide $x \in \mathcal{L}$ if, for any $i \in\{1, \ldots, k\}, y_{i} \in W_{x}$. Then, the probability of making an incorrect decision when $x \in \mathcal{L}$ is reduced to $\left(1-c_{\mathrm{yes}}\right)^{k}$.

BPP is the class of languages $\mathcal{L}$ where membership can be checked with two-sided error by a randomized, polynomial-time TM. Keeping the same notation as above, $\mathcal{L} \in \mathbf{B P P}$ if there are constants $c_{\text {yes }}$ and $c_{\text {no }}$ with $c_{\text {yes }}>c_{\text {no }}$, such that for all $x \in\{0,1\}^{n}$,

$$
\begin{aligned}
& x \in \mathcal{L} \Rightarrow \mu\left(W_{x}\right) \geq c_{\text {yes }}, \\
& x \notin \mathcal{L} \Rightarrow \mu\left(W_{x}\right) \leq c_{\text {no }}
\end{aligned}
$$


We can decide membership in $\mathcal{L}$ exactly the same as for $\mathbf{R P}$, but then there is a chance of making an incorrect decision both in the case when $x \in \mathcal{L}$ and when $x \notin \mathcal{L}$. The way to boost confidence in the decision is similar to that for RP: choose $y_{1}, \ldots, y_{k} \in_{R}\{0,1\}^{r}$ and decide $x \in \mathcal{L}$ if $y_{i} \in W_{x}$ for more than $k\left(c_{\text {no }}+c_{\text {yes }}\right) / 2$ of the $i \in\{1, \ldots, k\}$, and decide $x \notin \mathcal{L}$ otherwise.

\subsection{Complexity of unique solutions}

NP-hard problems often have many possible solutions. Would it make it easier if we were assured of a unique solution? Specifically, we say an algorithm $A \in \mathbf{R P}$ unique-solves an NP language $\mathcal{L}$ if, for all $x$, the output of $A$ is guaranteed to be "no" with high probability if $\left|W_{x}\right|=0$ and the output of $A$ is guaranteed to be "yes" with high probability if $\left|W_{x}\right|=1$. Notice there is no requirement on the output of $A$ if $\left|W_{x}\right|$ is neither 0 nor 1, i.e., $A$ can output anything in this case. Because of this, $A$ cannot be used directly to decide membership in $\mathcal{L}$. Valiant-Vazirani [39] nevertheless show that $A$ can be used indirectly to efficiently decide membership in $\mathcal{L}$. More specifically, ValiantVazirani [39] show that if there is an $A \in \mathbf{R P}$ that unique-solves some $\mathbf{N P}$-complete language $\mathcal{L}$ then $\mathbf{R P}=\mathbf{N P}$. The idea behind ValiantVazirani [39] follows.

Consider the following language CIRCUIT SAT: Given a circuit $C$ with an $r$-bit input, is there a $y \in\{0,1\}^{r}$ such that $C(y)=1$ ? A slightly more general NP-complete problem, $\Pi$, is the following: Given a circuit $C$ with an $r$-bit input, a function $h$ mapping $\{0,1\}^{r}$ to some set of values $T$, and a value $\alpha \in T$, is there an input $y$ to $C$ such that $C(y)=1$ and $h(y)=\alpha ?$

Theorem 3.1. If there is an algorithm $A \in \mathbf{R P}$ which unique-solves $\Pi$ then $\mathbf{R P}=\mathbf{N P}$.

Proof. We design an algorithm $B \in \mathbf{R P}$ that decides membership in CIRCUIT SAT based on $A$. On input a circuit $\mathrm{C}$ with an $r$-bit input, $B$ works as follows:

- Choose $k \in_{R}\{1, \ldots, r\}$. 
- Choose $s \in_{R} \mathcal{S}$, where $\mathcal{S}$ is the index set of the pairwise independent hash family (as in Section 1.2) that maps $U=\{0,1\}^{r}$ to $T=\{0,1\}^{k+1}$.

- Choose $\alpha \in R\{0,1\}^{k+1}$.

- Call $A\left(C, h_{s}, \alpha\right)$. Give the same answer as $A$.

Note that $\|s\|=\mathcal{O}(r)$, and so this reduction can be performed in random polynomial time. If $C$ is not satisfiable, then clearly $B$ will respond "no". If $C$ is satisfiable, then for some $k \in\{1, \ldots, r\}, 2^{k-1} \leq N \leq 2^{k}$, where $N$ is the number of satisfying assignments (witnesses) to $C$. With probability $1 / r$ we guessed this $k$ correctly.

Assume we have the correct $k$. Previously, we saw that for a table $T$, with $|T|=a N$, the expected number of colliding pairs $E[C] \leq N /(2 a)$. In our case, $2 \leq a \leq 4$, Thus, $E[C] \leq N / 4$. Hence, with probability at least $1 / 2$, at most $N / 2$ elements are paired and so at least $N / 2$ table entries are singletons. Assume this is the case. Since $|T| \leq 4 N, \operatorname{Pr}[$ there is a unique element that maps to $\alpha] \geq 1 / 8$.

Overall, the probability that we pick $\left(h_{s}, \alpha\right)$ so that $\left(C, h_{s}, \alpha\right)$ has a unique witness is at least $1 /(16 r)$. This can be boosted in the usual way. We thus have an $\mathbf{R P}$ algorithm for an NP-complete problem, implying $\mathbf{R P}=\mathbf{N P}$.

\section{3 $\quad$ BPP $\subseteq \sum_{2}$}

$\sum_{2}$ corresponds to languages $\mathcal{L}$ which can be written in the form:

$$
x \in \mathcal{L} \Longleftrightarrow \exists z, \forall w, q_{\mathcal{L}}(x, z, w)=1
$$

where $q_{\mathcal{L}}$ is a polynomial time predicate, and $\|z\|$ and $\|w\|$ are polynomial in $\|x\|$.

The following proofs are due to Sipser [36]. Consider the following class $\mathbf{B P} \mathbf{P}^{\prime}$, which is a very strong form $\mathbf{B P P}$ : For all $\mathcal{L} \in \mathbf{B P P}^{\prime}$ when $x \in \mathcal{L}$ then $\left|W_{x}\right|>2^{r-1}$ and when $x \notin \mathcal{L}$ then $\left|W_{x}\right| \leq 2^{(r-1) / 2}$. We can determine if $x \in \mathcal{L}$ for $\mathcal{L} \in \mathbf{B P P}^{\prime}$ as follows. Pick a $s \in_{R} \mathcal{S}$ where our hash family maps $\{0,1\}^{r} \rightarrow\{0,1\}^{r-1}$. For the case of $x \notin \mathcal{L}$, the table $T$ which $h_{s}$ maps to has $|T| \geq\left|W_{x}\right|^{2}$. We know from our previous analysis that in such a situation, $\operatorname{Pr}\left[h_{s}\right.$ is 1-to- 1$] \geq \frac{1}{2}$. When $x \in \mathcal{L}$, $\operatorname{Pr}\left[h_{s}\right.$ is 1 -to- 1$]=0$ (since the table is too small). Thus, we have a way 
of distinguishing the two situations. We can decide if $x \notin \mathcal{L}$ by the following,

$$
x \notin \mathcal{L} \Longleftrightarrow \begin{gathered}
\exists s \in \mathcal{S}, \forall y, y^{\prime} \in W_{x} \text { such that } y \neq y^{\prime}, \\
h_{s}(y) \neq h_{s}\left(y^{\prime}\right)
\end{gathered}
$$

This is a $\sum_{2}$ form of the complement of $\mathcal{L}$ (note that membership in $W_{x}$ takes only polynomial time to check). Therefore, $\overline{\mathbf{B P P}}^{\prime} \subseteq \sum_{2}$ and $\mathbf{B P P}^{\prime} \subseteq \Pi_{2}$. We now present the result of Sipser [36].

\section{Theorem 3.2. $\quad \mathrm{BPP} \subseteq \sum_{2}$.}

Proof. Consider the following version $\mathbf{B P P}^{\prime \prime}$ of BPP: For all $\mathcal{L} \in$ $\mathbf{B P P}^{\prime \prime}$, when $x \in \mathcal{L}$ then $\left|W_{x}\right|>\frac{1}{r} 2^{r}$ and when $x \notin \mathcal{L}$ then $\left|W_{x}\right| \leq \frac{1}{2 r^{2}} 2^{r}$. Using the usual amplification method, a language $\mathcal{L} \in \mathbf{B P P}$ can be easily reduced to a language $\mathcal{L}^{\prime} \in \mathbf{B} \mathbf{P} \mathbf{P}^{\prime \prime}$, and thus $\mathbf{B} \mathbf{P} \mathbf{P}^{\prime \prime}$ is equivalent to BPP.

We now show how to determine if $x \in \mathcal{L}$, where $\mathcal{L} \in \mathbf{B P P}^{\prime \prime}$. We use a table of size $t=\frac{1}{r^{2}} 2^{r}$. Pick $s_{1}, s_{2} \ldots, s_{r} \in_{R} \mathcal{S}$ where our pairwise independent hash family maps $\left.\{0,1\}^{r} \rightarrow\left[\frac{1}{r^{2}} 2^{r}\right]\right)$. Since we can't get a 1-to-1 mapping for one particular table, we consider for every witness in $W_{x}$ whether there is at least one mapping which isolates the witness. We notice $h$ isolates $y \in W_{x}$ iff for all $y^{\prime} \in W_{x}$ such that $y^{\prime} \neq y$, we have $h(y) \neq h\left(y^{\prime}\right)$.

Define an event $A$ : for all $y \in W_{x}$, there is an $i \in\{1, \ldots, r\}$ such that for all $y^{\prime} \in W_{x}$ such that $y^{\prime} \neq y, h_{s_{i}}(y) \neq h_{s_{i}}\left(y^{\prime}\right)$. We show that

$$
\begin{aligned}
& x \in \mathcal{L} \Rightarrow \operatorname{Pr}[A]=0 . \\
& x \notin \mathcal{L} \Rightarrow \operatorname{Pr}[A]>0 .
\end{aligned}
$$

Each $h_{s_{i}}$ can isolate at most $t$ elements. Hence, if $x \in \mathcal{L}$, the number of witnesses that can be isolated is $\leq t r=\frac{1}{r} 2^{r}<\left|W_{x}\right|$, and thus there must be some witnesses in $W_{x}$ that are not isolated by any of the $r$ hash functions, and thus $\operatorname{Pr}[A]=0$.

What if $x \notin \mathcal{L}$ ?

- Fix $i, y, y^{\prime} \cdot \operatorname{Pr}\left[h_{s_{i}}(y)=h_{s_{i}}\left(y^{\prime}\right)\right]=\frac{1}{t}$.

- Fix $i, y$. $\operatorname{Pr}\left[\exists y^{\prime} \in W_{x}, y^{\prime} \neq y, h_{s_{i}}(y)=h_{s_{i}}\left(y^{\prime}\right)\right] \leq \frac{\left|W_{x}\right|}{\ell} \leq \frac{1}{2}$. 
- Fix $y \cdot \operatorname{Pr}\left[\forall_{i}, \exists y^{\prime} \in W_{x}, y^{\prime} \neq y, h_{s_{i}}(y)=h_{s_{i}}\left(y^{\prime}\right)\right] \leq \frac{1}{2^{r}}$.

- $\operatorname{Pr}[A]=1-\operatorname{Pr}\left[\exists y \in W_{x}, \forall i \in\{1, \ldots, r\}, \exists y^{\prime} \in W_{x}, y^{\prime} \neq y\right.$, $\left.h_{s_{i}}(y)=h_{s_{i}}\left(y^{\prime}\right)\right] \geq 1-\frac{\left|W_{x}\right|}{2^{r}} \geq 1-\frac{1}{2 r^{2}}$.

- Thus, $\operatorname{Pr}[A]>0$.

Membership in language $\mathcal{L}$ can thus be summarized as:

$$
x \notin \mathcal{L} \Longleftrightarrow \begin{array}{r}
\exists h_{s_{1}}, \ldots, h_{s_{r}}, \forall y \in W_{x}, \exists i \in\{1, \ldots, r\} \\
\forall y^{\prime} \in W_{x}, y^{\prime} \neq y, h_{s_{i}}(y) \neq h_{s_{i}}\left(y^{\prime}\right)
\end{array}
$$

We've shown $\mathbf{B P P}=\mathbf{B P P}^{\prime \prime} \subseteq \sum_{4}$. Notice that the third quantifier $\exists i \in\{1, \ldots, r\}$ is of polynomial size. It is possible to eliminate this quantifier, and then the surrounding pair of "for all" quantifiers collapse together, giving $\mathbf{B P P} \subseteq \sum_{2}$.

\section{$3.4 \quad \mathrm{AM}=\mathrm{IP}$}

Goldwasser-Sipser [17] show that $\mathbf{A M}=\mathbf{I P}$, that is, public coins are as powerful as private coins in interactive protocols. To illustrate this we look at the graph non-isomorphism problem: $G N I=\left\{\left(G_{0}, G_{1}\right)\right.$ : graphs $G_{0}$ and $G_{1}$ are not isomorphic $\}$. To show that GNI $\in \mathbf{I P}$, we must exhibit a prover $P$ and verifier $V$ such that, for some pair of constants $c_{\text {yes }}$ and $c_{\text {no }}$ with $c_{\text {yes }}>c_{\text {no }}$ :

(1) For all $\left(G_{0}, G_{1}\right) \in$ GNI, $P$ causes $V$ to accept with probability at least $c_{\text {yes }}$.

(2) For all $\left(G_{0}, G_{1}\right) \notin$ GNI, every prover causes $V$ to accept with probability at most $c_{\text {no. }}$.

The coins of $V$ are kept private from the prover.

Let $G_{0}$ and $G_{1}$ both be graphs on $m$ nodes. The IP protocol proceeds as follows. $V$ picks a random permutation of $m$ nodes $\sigma \in_{R} \mathcal{S}_{m}$ and a random graph index $b \in_{R}\{0,1\}$ and sends $\sigma\left(G_{b}\right)$ to $P$, where $\sigma\left(G_{b}\right)$ is the graph indexed by $b$ with the nodes in the order specified by $\sigma$. $P$ then computes a bit $c \in\{0,1\}$, which is supposed to be the index of the graph sent by $V$, and sends $c$ to $V$. $V$ accepts iff $b=c$. 
If $\left(G_{0}, G_{1}\right) \in \mathrm{GNI}$, i.e., the graphs are not isomorphic, then $P$ can tell which graph is sent by $V$ and thus can compute $c$ correctly, so that $\operatorname{Pr}[V$ accepts $]=1=c_{\text {yes }}$. If the two graphs are isomorphic then, since the verifier sends a random permutation of the graph, the distribution on graphs received by the prover is the same whether $b=0$ or $b=1$, and since $b$ is chosen randomly the prover can answer correctly with probability $1 / 2$, and thus $\operatorname{Pr}[V$ accepts $]=1 / 2=c_{\text {no }}$.

Clearly, this protocol does not work if the coins used by the verifier to choose $b$ and $\sigma$ are public.

Now we look at an AM protocol for the same problem. Define $U$ to be the set of all $m$ vertex graphs. Define $W \subseteq U$ to be the set of all graphs that $V$ could have sent in the previous protocol, so $W=$ $\left\{\sigma\left(G_{b}\right): \sigma \in \mathcal{S}_{m}, b \in\{0,1\}\right\}$. Now,

$$
\begin{aligned}
& G_{0} \sim G_{1} \Rightarrow|W|=m !, \\
& G_{0} \nsim G_{1} \Rightarrow|W|=2(m !) .
\end{aligned}
$$

(This isn't always true, but something with a similar effect can be arranged), so the prover has to try to convince the verifier that the set $W$ is big. This is done by mapping the elements of $W$ into a table $T$ of size $4(m !)$ and looking at the probability that a random entry in $T$ is filled. The AM protocol proceeds as follows. $V$ randomly chooses $s \in_{R} \mathcal{S}$ and $\alpha \in_{R}\{1, \ldots, T\}$, and send $h_{s}$ and $\alpha$ to $P$. P computes $\sigma \in \mathcal{S}_{m}$ and $c \in\{0,1\}$ and sends to $V$ the graph $\sigma\left(G_{c}\right) . V$ accepts iff $h_{s}\left(\sigma\left(G_{c}\right)\right)=\alpha$. Note that unlike in the IP protocol described above, all the random bits used by $V$, i.e., $h_{s}$ and $\alpha$, are sent to $P$ and are thus public. We want to show that if $G_{0}$ is not isomorphic to $G_{1}$, then there is a fairly decent chance that the prover $P$ will be able to find a graph in $W$ (equivalently, a permutation $\sigma \in \mathcal{S}_{m}$ and $b \in\{0,1\}$ ) which is mapped to $\alpha$ by the hash function $h$. The following calculations apply to mapping a subset $W \subseteq U$ of size $N$ into a table $T$ of size $2 N$ (we are interested in $N=2(m !)$ ). Below we show that given an index $\alpha \in\{1, \ldots, 2 N\}, \operatorname{Pr}$ [at least one element in the size $N$ set is mapped to $\alpha] \geq 3 / 8=c_{\text {yes }}$. Define $E_{i}$ to be the event that element $i$ is mapped to the given $\alpha$. Then by inclusion-exclusion and using the fact that 


\section{Pairwise Independence and Complexity Classes}

the hash family is 2-universal, the above probability is:

$$
\begin{aligned}
\operatorname{Pr}\left[E_{1} \cup \ldots \cup E_{N}\right] & \geq \sum_{i=1}^{N} \operatorname{Pr}\left[E_{i}\right]-\sum_{i<j} \operatorname{Pr}\left[E_{i} \cap E_{j}\right] \\
& =\frac{N}{2 N}-\left(\begin{array}{c}
N \\
2
\end{array}\right) \frac{1}{4 N^{2}} \geq \frac{3}{8}
\end{aligned}
$$

Thus, if $x \in \mathcal{L}$ then $\operatorname{Pr}[V$ accepts $] \geq 3 / 8$. If $x \notin \mathcal{L}$, then the subset $W$ is $1 / 4$ the size of the table, so $\operatorname{Pr}[V$ accepts $] \leq 1 / 4=c_{\text {no }}$. The gap between these probabilities can be boosted in the usual way. 
A randomized algorithm computing some function errs with some positive probability. This error probability can be driven down to zero by simple repetition. For example consider an algorithm $A$ making one sided error when computing a Boolean function $f$ i.e., if $f(x)=0$, $A(x)$ is always 0 ; but if $f(x)=1$, then $A(x)=0$ with probability at most $1 / 2$. If we run $A$ several, say $k$, times on independent random sequences, and output 1 if any one of these trials output 1 , then we get an algorithm that errs with probability at most $2^{-k}$. Such a process, that drives down the error of a randomized algorithm is referred to as "amplification". The naive amplification described above reduces the error from $1 / 2$ to $2^{-k}$ but in the process needed a factor of $k$ more random bits than the original algorithm. The main quest of this chapter is to do better. We consider the amplification of a randomized algorithm without expending too much more randomness than the original algorithm.

\subsection{Deterministic amplification}

Let $\mathcal{L}$ be a $\mathbf{R P}$ language. A randomized $\mathbf{T M}$ can decide membership of $x \in\{0,1\}^{n}$ in $\mathcal{L}$ by choosing $y \in_{R}\{0,1\}^{r}$ and then checking if $y \in W_{x}$. 
As mentioned before, we can reduce the error probability of misclassification by choosing $y_{1}, \ldots, y_{k} \in_{R}\{0,1\}^{r}$, counting the number of these strings that fall in $W_{x}$ and basing the decision on the value of this number. If the $y_{i}$ 's are chosen independently, we need $k r$ random bits to achieve an error probability of $2^{-\mathcal{O}(k)}$. We'd like to use fewer random bits and still achieve a reduced error probability.

In this application, a pseudo-random generator is a deterministic TM, $G$, that takes a random seed $s$ and produces "pseudo-random" bits $G(s)=y_{1}, \ldots, y_{k}$, where each string is of length $r$. The algorithm is simply to test all $k$ of these strings and to conclude that $x \in \mathcal{L}$ if for any $i \in\{1, \ldots, k\} y_{i} \in W_{x}$ and $x \notin \mathcal{L}$ otherwise. Notice there is misclassification only when $x \in \mathcal{L}$ and $\left\{y_{1}, \ldots, y_{k}\right\} \subseteq \bar{W}_{x}$. We'll give several constructions.

\begin{tabular}{|l|l|l|}
\hline Generator & Random bits & Error \\
\hline Chor-Goldreich & $\mathcal{O}(r)$ & $\mathcal{O}(1 / k)$ \\
Impagliazzo-Zuckerman & $\mathcal{O}\left(r+k^{2}\right)$ & $2^{-\mathcal{O}(k)}$ \\
Nisan & $\mathcal{O}(r \lg k)$ & $2^{-\mathcal{O}(k)}$ \\
AKS & $r+\mathcal{O}(k)$ & $2^{-\mathcal{O}(k)}$ \\
\hline
\end{tabular}

For the Chor-Goldreich generator we show the result for $\mathcal{L} \in \mathbf{B P P}$. For all the other generators, we show the result for $\mathcal{L} \in \mathbf{R P}$. For these results, we assume $c_{\text {yes }} \geq 1 / 2$. Thus, the probability of a misclassification when $x \in \mathcal{L}$ is $<1 / 2$. All of these proofs can be extended to show an analogous result for $\mathcal{L} \in \mathbf{B P P}$ using exactly the same generator.

The results of the following exercise are due to Adleman [1] and Bennett-Gill [7]. These results show there is a polynomial size sample space that can be used to classify all $x \in\{0,1\}^{n}$ as either being in $\mathcal{L}$ or not, where $\mathcal{L} \in \mathbf{R P}$ or $\mathcal{L} \in \mathbf{B P P}$. The crucial property lacking from these results is that the sample space is not efficiently constructible. This property is the main point of the deterministic amplification constructions given in the following sections that reduce the number of random bits needed to find a witness with high probability.

Definition 4.1. ( $\mathbf{P} /$ poly) We say that $\mathcal{L} \in \mathbf{P} /$ poly if there is a polynomial time $\mathbf{T M} M(x, y)$ such that when $\|x\|=n$ then $\|y\|=r$, where $r$ is polynomial in $n$, with the following property: For each positive 
integer $n$, there is an "advice string" $y \in\{0,1\}^{r}$ with the property that, for all $x \in\{0,1\}^{n}$,

$$
\begin{aligned}
& x \in \mathcal{L} \Rightarrow M(x, y)=1, \\
& x \notin \mathcal{L} \Rightarrow M(x, y)=0 .
\end{aligned}
$$

We use the term "advice string" because, given the value of the advice string $y \in\{0,1\}^{r}$, it is easy to decide membership in $\mathcal{L}$ for all $x \in\{0,1\}^{n}$. Note that if it is possible to compute the value of the advice string $y \in\{0,1\}^{r}$ in $n^{\mathcal{O}(1)}$ time, then $\mathcal{L} \in P$. However, in general it may not be possible to compute the advice string in $n^{\mathcal{O}(1)}$ time. One way of thinking about a language $\mathcal{L} \mathbf{P} /$ poly is that membership in $\mathcal{L}$ can be decided in $n^{\mathcal{O}(1)}$ time with the aid of a polynomial amount of extra advice for each input length.

Exercise 4.2. Prove that $\mathbf{R P} \subseteq \mathbf{P} /$ poly and $\mathbf{B P P} \subseteq \mathbf{P} /$ poly.

\subsection{The Chor-Goldreich generator}

We first describe the generator due to Chor-Goldreich [10]. We show how this generator works for $\mathcal{L} \in \mathbf{B P P}$. We assume that $c_{\text {yes }} \geq 3 / 4$ and that $c_{\text {no }} \leq 1 / 4$. Let $\mathcal{S}$ be the index set for the pairwise independent hash family mapping $\{0,1\}^{r} \rightarrow\{0,1\}^{r}$, and let $s \in_{R} \mathcal{S}$. We let $G(s)=$ $h_{s}(1), \ldots, h_{s}(k)$; i.e., $y_{i}=h_{s}(i)$. Then the $y_{i}$ 's are uniformly distributed and pairwise independent. The algorithm concludes that $x \in \mathcal{L}$ if at least $k / 2$ of the strings $y_{1}, \ldots, y_{k}$ are in $W_{x}$ and $x \notin \mathcal{L}$ otherwise.

Theorem 4.3. The probability of misclassifying $x \in\{0,1\}^{n}$ with respect to membership in $\mathcal{L}$ is at most $4 / k$.

Proof. Define

$$
Z_{i}= \begin{cases}1 & \text { if } y_{i} \in W_{x} \\ 0 & \text { otherwise }\end{cases}
$$

The $Z_{i}$ 's are also identically distributed and pairwise independent with mean $\mu=\mu\left(W_{x}\right)$ and variance $\sigma^{2}=\mu(1-\mu) \leq 1 / 4$. Since the variance 
of $\sum_{i=1}^{k} Z_{i}$ is $k \sigma^{2}$, it follows (using the Chebyshev inequality) that

$$
\operatorname{Pr}\left[\left|\sum_{i=1}^{k} Z_{i}-\mu k\right|>\frac{k}{4}\right] \leq \frac{16 k \sigma^{2}}{k^{2}} \leq \frac{4}{k}
$$

\subsection{The Nisan generator}

We now describe the generator of Nisan [31]. Let $l=\log k$. Let $\mathcal{S}$ be the index set for the pairwise independent hash family $\left\{h_{s}\right\}$ mapping $\{0,1\}^{r} \rightarrow\{0,1\}^{r}$, and let $s_{1}, \ldots, s_{l} \in_{R} \mathcal{S}$. For every $y \in\{0,1\}^{r}$ let $G_{0}(y)=y$ and define inductively for $i \geq 1$,

$$
G_{i+1}\left(s_{1}, \ldots, s_{i+1}, y\right)=\left\langle G_{i}\left(s_{1}, \ldots, s_{i}, y\right), G_{i}\left(s_{1}, \ldots, s_{i}, h_{s_{i+1}}(y)\right)\right\rangle,
$$

where $\langle a, b\rangle$ denotes the concatenation of strings $a$ and $b$. For example, $G_{2}\left(s_{1}, s_{2}, y\right)=\left\langle y, h_{s_{1}}(y), h_{s_{2}}(y), h_{s_{1}}\left(h_{s_{2}}(y)\right)\right\rangle$. A more obvious way to visualize this generator is with a complete binary tree as shown in the following figure. A hash function is assigned to each level of this tree. The root of the tree is assigned the seed value $y$, and for any node $w$ on level $i \geq 1$ is assigned value $v_{w}$, where

$$
v_{w}= \begin{cases}v_{\text {parent }(w)} & \text { if } w \text { is the left child of parent }(\mathrm{w}) \\ h_{s_{l-i+1}}\left(v_{\text {parent }(w)}\right) & \text { otherwise }\end{cases}
$$

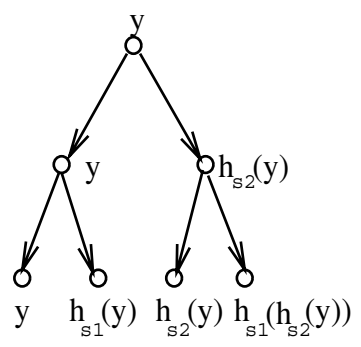

Notice $G_{l}\left(s_{1}, \ldots, s_{l}, y\right)$ is simply the concatenation of the strings on level $l$ of this tree.

Before proving this generator works we need the following technical lemma, which is also of independent interest. 
Hash Mixing Lemma: Let $\epsilon=2^{-r / 3}$ for some fixed parameter $r$. Then for all $A, B \subseteq\{0,1\}^{r}$, and for all but an $\epsilon$ fraction of $s \in \mathcal{S}$,

$$
\left|\operatorname{Pr}_{y \in \in_{R}\{0,1\}^{r}}\left[y \in A, h_{s}(y) \in B\right]-\operatorname{Pr}_{y, z \in_{R}\{0,1\}^{r}}[y \in A, z \in B]\right| \leq \epsilon
$$

Proof. We want to bound the number of $s \in \mathcal{S}$ such that

$$
\left|\operatorname{Pr}_{y \in \in_{R}\{0,1\}^{r}}\left[y \in A, h_{s}(y) \in B\right]-\mu(A) \mu(B)\right| \geq \epsilon
$$

This is exactly the number of $s \in \mathcal{S}$ such that

$$
\left|\operatorname{Pr}_{y \in R}\left[h_{s}(y) \in B\right]-\mu(B)\right| \geq \epsilon / \mu(A)
$$

Define the indicator random variable

$$
Z_{y}^{h_{s}}=\left\{\begin{array}{l}
1 \text { if } h_{s}(y) \in B \\
0 \text { otherwise }
\end{array}\right.
$$

A hash function $h_{s}$ is "bad" if

$$
\left|\sum_{y \in A} Z_{y}^{h_{s}}-\right| A|\mu(B)| \geq \frac{\epsilon|A|}{\mu(A)}=\epsilon 2^{r}
$$

By Chebyshev, and pairwise independence of our hash family,

$$
\operatorname{Pr}_{s \in \in_{R} \mathcal{S}}\left[\left|\sum_{y \in A} Z_{y}^{h_{s}}-\right| A|\mu(B)| \geq \epsilon 2^{r}\right] \leq \frac{\mu(B)|A|}{\epsilon^{2} 2^{2 r}}<\epsilon
$$

Theorem 4.4. If $x \in \mathcal{L}$ then $\operatorname{Pr}\left[G_{\ell}\left(s_{1}, \ldots, s_{\ell}, y\right) \subseteq \bar{W}_{x}\right] \leq \mu\left(\bar{W}_{x}\right)^{2^{\ell}}+$ $(\ell+2) \epsilon$ where $\epsilon=2^{-r / 3}$.

Proof. The $\epsilon \ell$ in the error term handles the hash functions that are "bad" for the Hash Mixing Lemma. Assume, for the moment, that $h_{s}$ for all $s \in \mathcal{S}$ satisfy the Hash Mixing Lemma. We show that

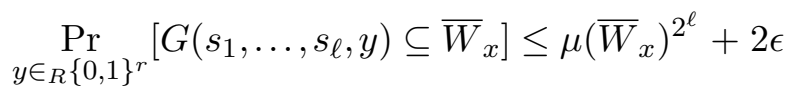

Inductively assume it is true for $\ell-1$. 
Let $A=B=\left\{y: G\left(s_{1}, \ldots, s_{\ell-1}, y\right) \subseteq \bar{W}_{x}\right\}$. These are the "bad" $y$, i.e., those $y$ for which we decide that $x \notin \mathcal{L}$ when in fact $x \in \mathcal{L}$. Now using the Hash Mixing Lemma.

$$
\begin{aligned}
\operatorname{Pr}\left[G\left(s_{1}, \ldots, s_{\ell}, y\right) \subseteq \bar{W}_{x}\right] & \leq \operatorname{Pr}\left[G\left(s_{1}, \ldots, s_{\ell-1}, y\right) \subseteq \bar{W}_{x}\right]^{2}+\epsilon \\
& \leq\left(\mu\left(\bar{W}_{x}\right)^{2^{\ell-1}}+2 \epsilon\right)^{2}+\epsilon \\
& \leq \mu\left(\bar{W}_{x}\right)^{2^{\ell}}+2 \epsilon
\end{aligned}
$$

where the first inequality holds by the Hash Mixing Lemma, and the second inequality holds by the induction hypothesis.

Each $s_{i}, i \in\{1, \ldots, \ell\}$, had an $\epsilon$ chance of being bad for the Hash Mixing Lemma, and so

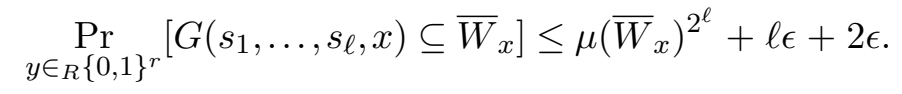

\subsection{The Impagliazzo-Zuckerman generator}

Let $\ell$ and $k$ be integer parameters. (A good setting is to make $k \approx$ $\ell \approx \sqrt{r}$ ). The generator described in Impagliazzo-Zuckerman [22] produces $k+1$ potential witnesses, each of length $r$, from only $3 r+k \ell$ random bits. Let $\mathcal{S}$ be the index set for the pairwise independent hash family $\left\{h_{s}\right\}$ mapping $\{0,1\}^{r} \rightarrow\{0,1\}^{r-\ell}$. The generator is defined by a function $G:\{0,1\}^{2 r} \times\{0,1\}^{r} \times\left\{\{0,1\}^{\ell}\right\}^{k} \rightarrow\left\{\{0,1\}^{r}\right\}^{k+1}$ :

$$
G\left(s, Y_{1}, Z_{1}, \ldots, Z_{k}\right) \mapsto\left(Y_{1}, Y_{2}, \ldots, Y_{k+1}\right)
$$

where $s \in_{R} \mathcal{S}, Y_{1} \in_{R}\{0,1\}^{r}$, and $Z_{i} \in_{R}\{0,1\}^{\ell}$, for $1 \leq i \leq k$. The $Y_{i}^{\prime}$ 's are defined by:

$$
Y_{i+1}=\left\langle h_{s}\left(Y_{i}\right), Z_{i}\right\rangle, \quad i=1, \ldots, k
$$

Theorem 4.5. If $x \in \mathcal{L}$ then,

$$
\operatorname{Pr}\left[G\left(s, Y_{1}, Z_{1}, \ldots, Z_{k}\right) \subseteq \bar{W}_{x}\right] \leq \mu\left(\bar{W}_{x}\right)^{k+1}+2^{1-\ell / 2}
$$


Several definitions and lemmas are needed to prove the theorem. Let $P$ and $Q$ be two probability distributions on a set $A$. The $L_{1}$-distance $\|\cdot\|_{1}$ and the $L_{2}$-distance $\|\cdot\|_{2}$ between $P$ and $Q$ are defined as

$$
\|P-Q\|_{1}=\sum_{i \in A}\left|P_{i}-Q_{i}\right|
$$

and

$$
\|P-Q\|_{2}=\left(\sum_{i \in A}\left(P_{i}-Q_{i}\right)^{2}\right)^{\frac{1}{2}}
$$

If $\Pi$ denotes the uniform distribution on a set $A$ then a distribution $P$ on the set $A$ is called $\epsilon$-uniform or $\epsilon$-quasi-random if $\|P-\Pi\|_{1} \leq \epsilon$.

The collision probability $c(P)$ of a probability distribution on a set $A$ is defined as

$$
c(P)=\operatorname{Pr}_{i, j \in P}[i=j]=\sum_{i \in A} P_{i}^{2}
$$

The next lemma states a simple condition for when a probability distribution is $\epsilon$-uniform.

Lemma 4.6. If $c(P) \leq\left(1+\epsilon^{2}\right) /|A|$ then $P$ is $\epsilon$-uniform on $A$.

Proof. By the Cauchy-Schwartz inequality, if $v \in \mathbf{R}^{n}$ then $\|v\|_{1} \leq$ $\sqrt{n}\|v\|_{2}$. Applying this to $P-\Pi$ yields

$$
|| P-\Pi\left|\|_{1}^{2} \leq\right| A\left|\sum_{i \in A}\left(\Pi_{i}-P_{i}\right)^{2}=\right| A \mid\left(\sum_{i \in A} \Pi_{i}^{2}-2 \sum_{i \in A} \Pi_{i} P_{i}+\sum_{i \in A} P_{i}^{2}\right) .
$$

Since $\Pi$ is the uniform distribution $\sum_{i \in A} \Pi_{i} P_{i}=1 /|A|$ and $\sum_{i \in A} \Pi_{i}^{2}=$ $1 /|A|$. By assumption $\sum_{i \in A} P_{i}^{2}=c(P) \leq\left(1+\epsilon^{2}\right) /|A|$.

Lemma 4.7. Let $\mathcal{S}$ be the index set of the pairwise independent hash family $\left\{h_{s}\right\}$ that maps $U$ to $T$. Let $P$ be the distribution $\left\langle s, h_{s}(x)\right\rangle$, where $s \in_{R} \mathcal{S}$ and $x \in_{R} W \subseteq U$ and let $A=\mathcal{S} \times T$. Then,

$$
c(P)=\frac{1+\frac{|T|}{|W|}}{|A|}
$$


Proof. Let $s, s^{\prime} \in_{R} \mathcal{S}$ and $x, x^{\prime} \in_{R} W$. Then,

$$
\begin{aligned}
c(P) & =\operatorname{Pr}\left[\left\langle s, h_{s}(x)\right\rangle=\left\langle s^{\prime}, h_{s^{\prime}}\left(x^{\prime}\right)\right\rangle\right] \\
& =\operatorname{Pr}\left[s=s^{\prime}\right] \operatorname{Pr}\left[h_{s}(x)=h_{s^{\prime}}\left(x^{\prime}\right) \mid s=s^{\prime}\right] \\
& =\operatorname{Pr}\left[s=s^{\prime}\right] \operatorname{Pr}\left[h_{s}(x)=h_{s}\left(x^{\prime}\right)\right] \\
& =\operatorname{Pr}\left[s=s^{\prime}\right]\left(\operatorname{Pr}\left[x=x^{\prime}\right]+\operatorname{Pr}\left[h_{s}(x)=h_{s^{\prime}}\left(x^{\prime}\right) \mid x \neq x^{\prime}\right]\right) \\
& =\frac{1}{\mathcal{S}}\left(\frac{1}{|W|}+\frac{1}{|T|}\right) \\
& =\frac{1}{|\mathcal{S}||T|}\left(1+\frac{|T|}{|W|}\right)
\end{aligned}
$$

The following lemma is from Impagliazzo-Levin-Luby [21].

Leftover-Hash-Lemma: Let $\mathcal{S}$ be the index set of the pairwise independent hash family $\left\{h_{s}\right\}$ that maps $U$ to $T$. For $s \in_{R} \mathcal{S}$ and $x \in_{R} W \subseteq$ $U$, the distribution $\left\langle s, h_{s}(x)\right\rangle$ is $\epsilon$-uniform, where $\epsilon=\sqrt{|T| /|W|}$.

Proof. Apply Lemma 4.7 and Lemma 4.6 in sequence.

Proof of Theorem: The proof is by induction on $k$ for a fixed value of $\ell$. Let error $_{k}=\operatorname{Pr}\left[G\left(s, Y_{1}, Z_{1}, \ldots, Z_{k}\right) \subseteq \bar{W}_{x}\right]$ be the error probability with respect to $k$. It is clear that error $_{0} \leq \mu\left(\bar{W}_{x}\right)$. For $k \geq 1$,

$$
\begin{aligned}
\operatorname{error}_{k} & =\operatorname{Pr}\left[Y_{1} \in \bar{W}_{x}\right] \operatorname{Pr}\left[G\left(s, Y_{1}, Z_{2}, \ldots, Z_{k}\right) \subseteq \bar{W}_{x} \mid Y_{1} \in \bar{W}_{x}\right] \\
& =\mu\left(\bar{W}_{x}\right) \operatorname{Pr}\left[G\left(s, Y_{2}, Z_{2}, \ldots, Z_{k}\right) \subseteq \bar{W}_{x} \mid Y_{1} \in \bar{W}_{x}\right]
\end{aligned}
$$

where $Y_{2}=\left\langle h_{s}\left(Y_{1}\right), Z_{1}\right\rangle$. Let $\epsilon=1 / \sqrt{2^{\ell} \mu\left(\bar{W}_{x}\right)}$. Then, it follows that

$$
\begin{aligned}
& \operatorname{Pr}\left[G\left(s, Y_{2}, Z_{2}, \ldots, Z_{k}\right) \subseteq \bar{W}_{x} \mid Y_{1} \in \bar{W}_{x}\right] \\
& \quad \leq \operatorname{Pr}\left[G\left(s, \hat{Y}_{2}, Z_{2}, \ldots, Z_{k}\right) \subseteq \bar{W}_{x}\right]+\epsilon,
\end{aligned}
$$

where $\hat{Y}_{2} \in_{R}\{0,1\}^{r}$. This is because the distribution $\left\langle s, h_{s}\left(Y_{1}\right)\right\rangle$ is $\epsilon$-uniform by the Leftover-Hash-Lemma, where $s \in_{R} \mathcal{S}$ and $Y_{1} \in_{R} \bar{W}_{x}$, and thus

$$
\left\|\left\langle s,\left\langle h_{s}\left(Y_{1}\right), Z_{1}\right\rangle, Z_{2}, \ldots, Z_{k}\right\rangle,\left\langle s, \hat{Y}_{2}, Z_{2}, \ldots, Z_{k}\right\rangle\right\|_{1} \leq \epsilon
$$


and this implies that the behavior of $G$ on these two distributions can differ by at most $\epsilon$.

The induction hypothesis implies that

$$
\operatorname{Pr}\left[G\left(s, \hat{Y}_{2}, Z_{2}, \ldots, Z_{k}\right) \subseteq \bar{W}_{x}\right] \leq \mu\left(\bar{W}_{x}\right)^{k}+2^{1-\ell / 2} .
$$

This implies

$$
\begin{aligned}
\operatorname{error}_{k} & \leq \mu\left(\bar{W}_{x}\right)\left(\mu\left(\bar{W}_{x}\right)^{k}+2^{1-\ell / 2}+1 / \sqrt{2^{\ell} \mu\left(\bar{W}_{x}\right)}\right) \\
& \leq \mu\left(\bar{W}_{x}\right)^{k+1}+2^{1-\ell / 2}
\end{aligned}
$$

where the last inequality uses $\mu\left(\bar{W}_{x}\right) \leq 1 / 2$ and $\sqrt{\mu\left(\bar{W}_{x}\right)} \leq 1$.

\subsection{The expander mixing Lemma}

This section will use expander graphs (see [20] for a survey), a combinatorial object of extreme importance in many areas of theoretical computer science and mathematics, and particularly in derandomization.

Let $G=(U, E)$ be a $d$-regular undirected graph with $n$ nodes $(|U|=$ $n$ ). The adjacency matrix of $G$ is a symmetric $n \times n$-matrix $M$ with

$$
M(i, j)=\left\{\begin{array}{l}
0(i, j) \notin E \\
1(i, j) \in E
\end{array} .\right.
$$

Every such matrix has an orthonormal basis of eigenvectors. Let these eigenvectors for $M$ be the vectors $\mathbf{r}_{0}, \ldots, \mathbf{r}_{n-1} \in \mathbf{R}^{n}$ with the corresponding eigenvalues $\lambda_{0}, \ldots, \lambda_{n-1} \in \mathbf{R}$. Define $\delta_{i, j}=1$ if $i=j$ and $\delta_{i, j}=0$ if $i \neq j$. We let · denote multiplication of matrices over the reals. Whenever a vector is involved in a multiplication, we use the convention that it is a row vector if it is to the left of · and a column vector if it is to the right of $\cdot$. Thus, if $a$ and $b$ are equal length vectors then $a \cdot b$ denotes the inner product of $a$ and $b$ over the reals. We have for all $0 \leq i, j \leq n-1$ :

$$
\begin{aligned}
\mathbf{r}_{i} \cdot \mathbf{r}_{j} & =\delta_{i, j} \\
M \cdot \mathbf{r}_{i} & =\lambda_{i} \mathbf{r}_{i}
\end{aligned}
$$


Every row of $M$ consists of $d$ ones and $n-d$ zeros. Hence the vector of ones in all components (denoted $\overline{1} \in \mathbf{R}^{n}$ ) is an eigenvector of $M$ corresponding to the eigenvalue $d$. Furthermore all eigenvalues are realvalued and no larger than $d$. Without loss of generality, we assume $\mathbf{r}_{0}=\overline{1} / \sqrt{n}$ and $\lambda_{0}=d$. Let

$$
\lambda=\max _{1 \leq i \leq n-1}\left|\lambda_{i}\right|
$$

denote the second largest eigenvalue, that is the maximum factor by which a vector orthogonal to $\mathbf{r}_{0}$ is stretched when multiplied by $M$.

Multiplication of a vector $\mathbf{z} \in \mathbf{R}^{n}$ with $M$ can easily be expressed using the eigenvectors and eigenvalues: Setting $\gamma_{i}=\mathbf{z} \cdot \mathbf{r}_{i}$ we have

$$
\begin{aligned}
\mathbf{z} & =\sum_{0 \leq i \leq n-1} \gamma_{i} \mathbf{r}_{i} \\
\text { and } M \cdot \mathbf{z} & =\sum_{0 \leq i \leq n-1} \lambda_{i} \gamma_{i} \mathbf{r}_{i} .
\end{aligned}
$$

For two sets $A, B \subset U$ denote the set of (directed) edges from $A$ to $B$ in $G$ by $E(A, B)=\{(v, w) \in A \times B:(v, w) \in E\}$. Fixing $A$ and an integer $b$, and picking a subset $B$ of $U$, of size $b$ uniformly at random, the expected size of $E(A, B)$ is $\frac{d|A| b}{n}$. The following lemma states that for any sets $A, B$ the size of $E(A, B)$ is close to its expectation, where "close" depends on the value of $\lambda$. While similar statements were known before, this convenient form appears first in [6]

Expander Mixing Lemma: For all $A, B \subseteq U$ it holds that

$$
|| E(A, B)\left|-\frac{d|A||B|}{n}\right| \leq \lambda \sqrt{|A||B|} \leq \lambda n .
$$

Proof. Let $\mathcal{X}_{A} \in \mathbf{R}^{n}$ denote the indicator vector of $A$, i.e. a "one" is at the position corresponding to a vertex $v \in A$ and a "zero" for $v \notin A$. $\mathcal{X}_{B}$ is the corresponding vector for $B$. Set

$$
\begin{aligned}
\alpha_{i} & =\mathcal{X}_{A} \cdot \mathbf{r}_{i} \\
\beta_{i} & =\mathcal{X}_{B} \cdot \mathbf{r}_{i}
\end{aligned}
$$


Then $\alpha_{0}=|A| / \sqrt{n}$ and $\beta_{0}=|B| / \sqrt{n}$, and we have

$$
\begin{aligned}
|E(A, B)| & =\sum_{i \in A, j \in B} M(i, j) \\
& =\mathcal{X}_{A} \cdot M \cdot \mathcal{X}_{B} \\
& =\left(\sum_{0 \leq i \leq n-1} \alpha_{i} \mathbf{r}_{i}\right) \cdot\left(\sum_{0 \leq j \leq n-1} \lambda_{j} \beta_{j} \mathbf{r}_{j}\right) \\
& =\sum_{0 \leq i \leq n-1} \lambda_{i} \alpha_{i} \beta_{i} \\
& =\frac{d|A||B|}{n}+\sum_{1 \leq i \leq n-1} \lambda_{i} \alpha_{i} \beta_{i} \\
\Longrightarrow|| E(A, B)\left|-\frac{d|A||B|}{n}\right| & \leq \lambda \sum_{0 \leq i \leq n-1}\left|\alpha_{i} \beta_{i}\right| \\
& \leq \lambda\|\alpha\|_{2}\|\beta\|_{2} \\
& =\lambda\left\|\mathcal{X}_{A}\right\|_{2}\left\|\mathcal{X}_{B}\right\|_{2} \\
& =\lambda \sqrt{|A \| B|}
\end{aligned}
$$

Another way to state the Expander Mixing Lemma is that for all $A$, $B \subseteq U$,

$$
\left|\operatorname{Pr}_{x, s}\left[x \in A, e_{s}(x) \in B\right]-\operatorname{Pr}_{x, y}[x \in A, y \in B]\right| \leq \frac{\lambda}{d},
$$

where in the first random experiment $x$ is a uniformly chosen node, $s \in_{R}\{1, \ldots, d\}$, and $e_{s}(x)$ is the neighbor of $x$ indexed by $s$, whereas in the second experiment $x$ and $y$ are two independently and uniformly chosen nodes. Note the resemblance with the Hash Mixing Lemma, where $e_{s}(x)$ is substituted by $h_{s}(x)$.

There are explicit constructions of symmetric matrices with a small second largest eigenvalue $\lambda$, corresponding to graphs with good expansion properties. For all integers $n^{\prime}$ and $d^{\prime}$ there is an explicit construction of an $n$ node $d$-regular graph $G$ with $n^{\prime} \leq n \leq 2 n^{\prime}, d^{\prime} \leq d \leq 2 d^{\prime}$ and $\lambda \leq d^{9 / 10}$. (For example, see either Lubotzky-Phillips-Sarnak [27] or Margulis [29], and the survey [20].) For every node $x \in U$ and integer 
$s \in\{1, \ldots, d\}$ the $s$-th neighbor $e_{s}$ of $x$ in $G$ can be computed in logarithmic space. (To simplify the presentation we assume in the sequel that we can construct expanders for all values of $n$ and $d$.)

\subsection{The Karp-Pippenger-Sisper generator}

The Karp-Pippenger-Sipser [25] generator uses the explicit construction of expanders: The set $\{0,1\}^{r}$ is identified with the nodes of a $2^{r}$ node $k$-regular expander with $\lambda \leq k^{9 / 10}$. The seed to the generator $G$ is a string $z \in_{R}\{0,1\}^{r}$, and $G(z)$ produces $y_{1}, \ldots, y_{k}$, which are the $k$ neighbors of $z$ in the expander graph. Thus, this scheme uses exactly $r$ random bits.

Theorem 4.8. If $x \in \mathcal{L}$ then

$$
\operatorname{Pr}\left[\left\{y_{1}, \ldots, y_{k}\right\} \subseteq \bar{W}_{x}\right] \leq 2 k^{-1 / 10} .
$$

Proof. Let $A \subseteq\{0,1\}^{r}$ be the set of nodes $z$ with the property that all neighbors of $z$ are in $\bar{W}_{x}$. Thus

$$
\operatorname{Pr}\left[\left\{y_{1}, \ldots, y_{k}\right\} \subseteq \bar{W}_{x}\right]=|A| / 2^{r} .
$$

From $E\left(A, W_{x}\right)=\emptyset$ and the Expander Mixing Lemma it follows that

$$
\begin{aligned}
& \frac{k|A|\left|W_{x}\right|}{2^{r}} \leq \lambda 2^{r} \\
& \Longrightarrow \frac{|A|}{2^{r}} \leq \frac{\lambda 2^{r}}{k\left|W_{x}\right|} \\
& \leq 2 k^{-1 / 10} \quad\left(\text { since }\left|W_{x}\right| \geq 2^{r-1}\right)
\end{aligned}
$$

\subsection{The Ajtai-Komlós-Szemerédi generator}

Ajtai-Komlós-Szemerédi [3] show how to simulate a randomized $\log$-space computation using $O\left(\frac{(\log n)^{2}}{\log \log n}\right)$ random bits by a deterministic log-space computation. Cohen-Wigderson [11] and NisanZuckerman [33] observe that the AKS generator can also be used for amplification: Let $n=2^{r}$ and identify the set $\{0,1\}^{r}$ with the nodes 
of a $d$-regular $n$-node expander graph $G$. Set $d$ to some constant value so that $\lambda \leq d / 4$ can be achieved. Choose the nodes $y_{1}, \ldots, y_{k}$ as the nodes visited on a random walk of length $k$ starting at a random node $z \in_{R}\{0,1\}^{r}$. The random walk is determined by the starting point $z$ and integers $i_{j} \in\{1, \ldots, d\}$ for $j \in\{1, \ldots, k\}$ describing which edge to use in $j$-th step of the walk. Thus, $y_{1}$ is the $i_{1}$-th neighbor of $z$ and, for $j \geq 2, y_{j}$ is the $i_{j}$-th neighbor of $y_{j-1}$. The number of random bits used is $r+k \log d=r+\mathcal{O}(k)$.

Theorem 4.9. If $x \in \mathcal{L}$ then

$$
\operatorname{Pr}\left[\left\{y_{1}, \ldots, y_{k}\right\} \subseteq \bar{W}_{x}\right]=2^{-\Theta(k)} .
$$

Proof. To bound the error probability we describe the probability distribution after subsequent steps of the random walk by an $n$-dimensional vector. Let $\mathbf{p}_{0}$ be the vector describing the initial distribution, i.e., the distribution of $z$, which is $\mathbf{p}_{0}(v)=1 / n$ for all $v \in\{0,1\}^{r}$. Let $M$ be the adjacency matrix of $G$ and set $\hat{M}=M / d$. Thus, the distribution after the first step, i.e., the distribution of $y_{1}$, is $\mathbf{p}_{1}=M \cdot \mathbf{p}_{0}$. We are interested in the probability that all nodes $y_{1}, \ldots, y_{k}$ are contained in $\bar{W}_{x}$. The probability of $y_{1} \in \bar{W}_{x}$ is obtained by cancelling out the components of $\mathbf{p}_{1}$ corresponding to nodes in $W_{x}$ and summing up the other components. Let $P_{\bar{W}_{x}}$ be the diagonal matrix with ones in the positions corresponding to elements of $\bar{W}_{x}$ and zeros elsewhere. Then

$$
\operatorname{Pr}\left[y_{1} \in \bar{W}_{x}\right]=\left\|P_{\bar{W}_{x}} \cdot \hat{M} \cdot \mathbf{p}_{0}\right\|_{1}
$$

Continuing this process yields

$$
\operatorname{Pr}\left[y_{1} \in \bar{W}_{x} \wedge \cdots \wedge y_{k} \in \bar{W}_{x}\right]=\left\|\left(P_{\bar{W}_{x}} \cdot \hat{M}\right)^{k} \cdot \mathbf{p}_{0}\right\|_{1} .
$$

For any vector $\mathbf{z}=\sum_{0 \leq i \leq n-1} \gamma_{i} \mathbf{r}_{i}$ we have

$$
\begin{aligned}
\left\|P_{\bar{W}_{x}} \cdot \hat{M} \cdot \mathbf{z}\right\|_{2} & \leq\left\|P_{\bar{W}_{x}} \cdot \hat{M} \cdot \gamma_{0} \mathbf{r}_{0}\right\|_{2} \\
& +\left\|P_{\bar{W}_{x}} \cdot \hat{M} \cdot \sum_{1 \leq i \leq n-1} \gamma_{i} \mathbf{r}_{i}\right\|_{2}
\end{aligned}
$$




\section{Recycling Randomness}

Using $\hat{M} \cdot r_{0}=r_{0}$ and replacing $P_{\bar{W}_{x}}$ by $I$, we continue the inequalities as follows:

$$
\begin{aligned}
& \leq\left\|P_{\bar{W}_{x}} \cdot \gamma_{0} \mathbf{r}_{0}\right\|_{2}+\left\|\hat{M} \cdot \sum_{1 \leq i \leq n-1} \gamma_{i} \mathbf{r}_{i}\right\|_{2} \\
& \leq \sqrt{\mu\left(\bar{W}_{x}\right)}\left\|\gamma_{0} \mathbf{r}_{0}\right\|_{2}+\left\|\sum_{1 \leq i \leq n-1} \frac{\lambda_{i}}{d} \gamma_{i} \mathbf{r}_{i}\right\|_{2} \\
& \leq \sqrt{\mu\left(\bar{W}_{x}\right)}\left\|\gamma_{0} \mathbf{r}_{0}\right\|_{2}+\frac{\lambda}{d}\left\|\sum_{1 \leq i \leq n-1} \gamma_{i} \mathbf{r}_{i}\right\|_{2} \\
& \leq\left(\sqrt{\mu\left(\bar{W}_{x}\right)}+\frac{\lambda}{d}\right)\|\mathbf{z}\|_{2}
\end{aligned}
$$

(The last inequality is based on the fact that both $\gamma_{0} \mathbf{r}_{0}$ and $\sum_{1 \leq i \leq n-1} \gamma_{i} \mathbf{r}_{i}$ are both projections of $z$.) Applying this inequality to (1) and using Cauchy-Schwarz we are able to bound the error probability:

$$
\begin{aligned}
\operatorname{Pr}\left[y_{1} \in \bar{W}_{x} \wedge \cdots \wedge y_{k} \in \bar{W}_{x}\right] & \leq \sqrt{n}\left\|\left(P_{\bar{W}_{x}} \cdot \hat{M}\right)^{k} \cdot \mathbf{P}_{0}\right\|_{2} \\
& \leq \sqrt{n}\left(\sqrt{\mu\left(\bar{W}_{x}\right)}+\frac{\lambda}{d}\right)^{k}\left\|\mathbf{P}_{0}\right\|_{2} \\
& \leq \sqrt{n}(\sqrt{1 / 2}+1 / 4)^{k} / \sqrt{n} \\
& =2^{-\Theta(k)} .
\end{aligned}
$$




\section{Pseudo-Random Generators}

In this chapter we introduce one-way functions and pseudo-random generators, and show how to construct a pseudo-random generator from a one-way function. The reason for interest in these cryptographic functions and for the reduction from a one-way function to a pseudo-random generator is that there are a lot of natural examples of functions that seem to be one-way, while pseudo-random generators are extremely useful in the design of cryptographic protocols and in derandomization of algorithms.

\subsection{One-way functions}

Intuitively, a one-way function is a function that is easy to compute but hard for any time-bounded adversary to invert on a random input. To gauge the success of an adversary in breaking a cryptographic function, we use the following measure.

Definition 5.1. (time/success ratio) The time/success ratio of an adversary for breaking a cryptographic function is $T(n) / \delta(n)$, where $T(n)$ is the run time of the adversary and $\delta(n)$ is the success probability 
of the adversary with respect to inputs parameterized by $n$. The definition of the success probability depends on the cryptographic function.

Definition 5.2. (one-way function) Let $f(x)$ be a function computable in time polynomial in $\|x\|$. The success probability (inverting probability) of adversary $A$ for $f$ is

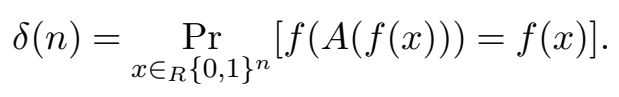

Then, $f$ is a $\mathbf{S}(n)$-secure one-way function if every $A$ has time/success ratio at least $\mathbf{S}(n)$.

Definition 5.3. (one-way permutation) Exactly the same as the definition of a one-way function, except that $\|f(x)\|=\|x\|$ and $f$ as a function of $x \in\{0,1\}^{n}$ is a permutation.

\subsubsection{Examples of conjectured one-way functions}

Here are some natural examples that may eventually be proven to be one-way functions. Plenty of others can be found in the literature. In the following, $p$ and $q$ are primes of length $n$.

Factoring problem: Define $f(p, q)=p q$. It is possible to compute $p q$ given $p$ and $q$ in $n^{\mathcal{O}(1)}$ time. However, there is no known polynomialtime function that on input $p q$ can produce $p$ and $q$ on average for randomly chosen pairs of primes $\langle p, q\rangle$

Discrete $\log$ problem: Let $g$ be a generator of $\mathcal{Z}_{p}^{*}$, i.e., for all $y \in \mathcal{Z}_{p}^{*}$, there is a unique $x \in \mathcal{Z}_{p-1}$ such that $g^{x}=y \bmod p$. Given $p, g$ and $x \in \mathcal{Z}_{p-1}$, define $f(p, g, x)=\left\langle p, g, g^{x} \bmod p\right\rangle$. It is possible to compute $g^{x} \bmod p$ given $p, g$ and $x$ in $n^{\mathcal{O}(1)}$ time. The discrete log function is a permutation as a function of $x$, i.e., the unique inverse of $f(p, g, x)$ is $\langle p, g, x\rangle$. The values of $p$ and $g$ are not necessarily chosen randomly. The prime $p$ is selected to have special properties which seem in practice to make the discrete log function hard to invert. An example of such a 
property is that $p$ is selected so that $p-1$ has some fairly large prime divisors. For a large class of primes $p$ and generators $g$ there is no known polynomial-time function that on input $p, g$ and $g^{x} \bmod p$ can produce $x$ on average for $x \in_{R} \mathcal{Z}_{p-1}$.

Root extraction problem: Given $p, q, e \in \mathcal{Z}_{p-1}$ and $y \in \mathcal{Z}_{p}$, define $f(p, q, e, y)=\left\langle p q, e, y^{e} \bmod p q\right\rangle$. It is possible to compute $y^{e} \bmod p q$ given $p q, e$ and $y$ in $n^{\mathcal{O}(1)}$ time. To make the inversion problem hard, it is important that the factorization of the modulus is not part of the output, because given the factorization an inverse can be found in $n^{\mathcal{O}(1)}$ time. The value of the exponent $e$ is not necessarily chosen randomly. For example, if $e=2$ then the problem is to extract square roots, and this still seems to be a hard problem on average. There is no known polynomial-time function that on input $p q, e$ and $y^{e} \bmod p q$ can produce an $y^{\prime} \in \mathcal{Z}_{p}$ such that $y^{\prime e}=y^{e} \bmod p q$ when $p$ and $q$ are randomly chosen according to a distribution for which factoring is hard and $y \in_{R} \mathcal{Z}_{p}$. There is a strong connection between this problem when $e=2$ and the factoring problem.

Subset sum problem: Let $a \in\{0,1\}^{n}$ and $b \in\{0,1\}^{n \times n}$. Given $a$ and $b$, define $f(a, b)=\left\langle\sum_{i=1}^{n} a_{i} \cdot b_{i}, b\right\rangle$, where $a_{i} \in\{0,1\}$ and $b_{i}$ is an $n$-bit integer in this expression and where the sum is over the integers. It is possible to compute $\sum_{i=1}^{n} a_{i} \cdot b_{i}$ given $a$ and $b$ in $n^{\mathcal{O}(1)}$ time. However, there is no known polynomial-time function that on input $\sum_{i=1}^{n} a_{i} \cdot b_{i}$ and $b$ can produce $a^{\prime} \in\{0,1\}^{n}$ such that $\sum_{i=1}^{n} a_{i}^{\prime} \cdot b_{i}=\sum_{i=1}^{n} a_{i} \cdot b_{i}$ on average when $a \in_{R}\{0,1\}^{n}$ and $b \in_{R}\{0,1\}^{n \times n}$.

Exercise 5.4. Let $A \in_{R}\{0,1\}^{n}$ and let $B \in_{R}\{0,1\}^{n \times(n+1)}$. Prove that the probability

$$
f(A, B)=\left\langle\sum_{i=1}^{n} A_{i} \cdot B_{i}, B\right\rangle
$$

has a unique inverse is lower bounded by a constant strictly greater than zero independent of $n$. Note that in contrast to the previous definition where $\left\|B_{i}\right\|=n$, here $\left\|B_{i}\right\|=n+1$. 


\subsection{Hidden Bit Theorem}

The main result of these sections is the construction of a pseudo-random generator from any one-way permutation. In this section, we present the main technical content of this reduction, the Hidden Bit Theorem, which is due to Goldreich-Levin [15].

There are several technical parts in the reduction from any one-way permutation $f$ to a pseudo-random generator $g$. Intuitively, the Hidden Bit Theorem is the part that transforms the one-wayness of $f$ into a bit $b$ such that: (1) $b$ is completely determined by information that is available to any adversary; (2) nevertheless $b$ looks random to any appropriately time-restricted adversary. It is from this bit $b$ that the generator $g$ eventually derives its pseudo-randomness. The guarantee from the reduction is that any successful adversary for distinguishing the output of $g$ from a truly random string can be converted into an adversary for predicting $b$, which in turn can be converted into an adversary for inverting $f$.

The definition of a computationally hidden but statistically meaningful bit and the realization of its importance as a basic building block for cryptographic constructions is from Blum-Micali [8].

The construction of a hidden bit using the inner product bit, the Hidden Bit Theorem and the Hidden Bit Technical Theorem are all from Goldreich-Levin [15]. The simpler proof given here of Hidden Bit Technical Theorem is due to C. Rackoff, R. Venkatesan and L. Levin, inspired by Alexi-Chor-Goldreich-Schnorr [4].

Definition 5.5. (inner product bit is hidden) Let $f(x)$ be a polynomial-time computable function. Let $x \in\{0,1\}^{n}$ and $z \in\{0,1\}^{n}$. Then, the inner product bit of $f(x)$ is $x \odot z$. The success probability (prediction probability) of adversary $A$ for the inner product bit of $f$ is

$\delta(n)=\operatorname{Pr}_{x, z \in_{R}\{0,1\}^{n}}[A(f(x), z)=x \odot z]-\operatorname{Pr}_{x, z \in_{R}\{0,1\}^{n}}[A(f(x), z) \neq x \odot z]$.

Then, the inner product bit of $f$ is a $\mathbf{S}(n)$-secure if every $A$ has time/success ratio at least $\mathbf{S}(n)$. 
Hidden Bit Theorem: If $f$ is a one-way function then the inner product bit of $f$ is hidden. In particular, there is a TM $M$ such that if $A$ is an adversary with time/success ratio $\mathbf{S}(n)$ for predicting the inner product bit then $M^{A}$ is an adversary with time/success ratio $\mathbf{S}(n)^{c}$ for inverting $f$ for some constant $c>0$. $\left(M^{A}\right.$ denotes $M$ making calls to the adversary $A$.)

Proof. Suppose there is an adversary $A$ for the inner product bit of $f$ with success probability $\delta(n)$ and run time $T(n)$. We describe a TM $M$ such that $M^{A}$ is an adversary for $f$ as a one-way function.

For $x \in\{0,1\}^{n}$ define

$$
\delta_{x}^{A}=\operatorname{Pr}_{z \in_{R}\{0,1\}^{n}}[A(f(x), z)=x \odot z]-\operatorname{Pr}[A(f(x), z) \neq(x \odot z)] .
$$

Let $X \in \in_{R}\{0,1\}^{n}$. Because, for any $x \in\{0,1\}^{n},\left|\delta_{x}^{A}\right| \leq 1$ and because $E_{X}\left[\delta_{X}^{A}\right]=\delta(n)$, it follows that $\operatorname{Pr}_{X}\left[\delta_{X}^{A} \geq \delta(n) / 2\right] \geq \delta(n) / 2$. The TM $M$ we describe below has the property that if $\delta_{x}^{A} \geq \delta(n) / 2$ then $M^{A}$ on input $f(x)$ succeeds in producing an $x^{\prime}$ such that $f\left(x^{\prime}\right)=f(x)$ with probability at least $1 / 2$. From this it follows that the inverting probability of $M^{A}$ for $f$ is at least $\delta(n) / 4$.

Suppose the input to $M^{A}$ is $f(x)$, where $\delta_{x}^{A} \geq \delta(n) / 2$. Let $S$ be the TM described below in the Hidden Bit Technical Theorem (subsection 5.2.1) and let $B(z)=A(f(x), z)$. The first step of $M^{A}$ is to run $S^{B}$ with input $\delta=\delta(n) / 2$. When $S$ makes an oracle query to $B$ with input $z, M$ runs $A$ on input $\langle f(x), z\rangle$ and returns the answer $B(z)=A(f(x), z)$ to $S$. Because $\delta_{z}^{A} \geq \delta(n) / 2$, by the Hidden Bit Technical Theorem, $x$ is in the list $\mathcal{L}$ produced by $S^{B}$ with probability at least $1 / 2$. The final step of $M^{A}$ to do the following for all $x^{\prime} \in \mathcal{L}$ : Compute $f\left(x^{\prime}\right)$ and if $f\left(x^{\prime}\right)=f(x)$ then output $x^{\prime}$.

The success probability of $M^{A}$ for inverting $f(X)$ is at least $\delta(n) / 4$. From the Hidden Bit Technical Theorem, it is not hard to see that the running time of $M^{A}$ is dominated by the running time of $S$ making queries to $A$ to produce the list $\mathcal{L}$, which is $\mathcal{O}\left(n^{3} T(n) / \delta(n)^{4}\right)$, where $T(n)$ is the running time of $A$. Thus, the time/success ratio of $M^{A}$ is $\mathcal{O}\left(n^{3} T(n) / \delta(n)^{5}\right)$. 


\subsubsection{Generalized inner product space}

For the proof of the Hidden Bit Technical Theorem we use the following generalization of the Inner Product Space (Section 2.4).

Generalized Inner Product Space: Let $l=[\log (m+1)]$. The sample space is $\mathcal{S}=\{0,1\}^{n \times l}$ and the distribution on sample points is $v \in_{R} \mathcal{S}$. For all $j \in\{0,1\}^{l}$, define random variable

$$
T_{j}(v)=v \odot j .
$$

It can be verified that $T_{1}(v), \ldots, T_{m}(v)$ are uniformly distributed on $\{0,1\}^{n}$ and pairwise independent.

Hidden Bit Technical Theorem: Let $B(z)$ be a TM which runs in time polynomial in $n$, and for each $x \in\{0,1\}^{n}$ define

$$
\delta_{x}^{B}=\operatorname{Pr}_{z \in \in_{R}\{0,1\}^{n}}[B(z)=x \odot z]-\operatorname{Pr}_{z \in_{R}\{0,1\}^{n}}[B(z) \neq x \odot z] .
$$

There is a TM $S$ such that for any $B, S^{B}$ on input $\delta>0$ produces a list $\mathcal{L} \subseteq\{0,1\}^{n}$ with the following property: For all $x \in\{0,1\}^{n}$, if $\delta_{x}^{B} \geq \delta$ then $x \in \mathcal{L}$ with probability at least $1 / 2$, where this probability is with respect to the random bits used by TM $S^{B}$. The running time of $S^{B}$ is $\mathcal{O}\left(n^{3} T / \delta^{4}\right)$, where $T$ is the running time of $B$.

Proof. For the proof, we find it convenient to consider bits as being $\{1,-1\}$-valued instead of $\{0,1\}$-valued. For $b \in\{0,1\}$, we let $\bar{b}=(-1)^{b} \in$ $\{1,-1\}$.

Fix $x \in\{0,1\}^{n}$ such that $\delta_{x}^{B} \geq \delta$. We can write

$$
\delta_{x}^{B}=\mathrm{E}_{z \in_{R}\{0,1\}^{n}}[\overline{B(z)} \cdot \overline{x \odot z}] .
$$

For all $i=1, \ldots, n$, let $e_{i} \in\{0,1\}^{n}$ be the $i$ th unit vector, i.e. $\left\langle 0^{i-1}, 1,0^{n-i}\right\rangle$, and let

$$
\mu_{i}=\delta_{x}^{B} \cdot \overline{x_{i}}
$$

It follows that

$$
\mathrm{E}_{z \in_{R}\{0,1\}^{n}}\left[\overline{B(z)} \cdot \overline{x \odot\left(e_{i} \oplus z\right)}\right]=\mu_{i} .
$$


This is because $\odot$ distributive over $\oplus$ implies that

$$
\overline{x \odot\left(e_{i} \oplus z\right)}=\overline{x \odot e_{i}} \cdot \overline{x \odot z}
$$

and because $\overline{x \odot e_{i}}=\overline{x_{i}}$, and thus

$$
\overline{B(z)} \cdot \overline{x \odot\left(e_{i} \oplus z\right)}=\overline{B(z)} \cdot \overline{x \odot z} \cdot \overline{x_{i}} .
$$

Setting $z^{\prime}=e_{i} \oplus z$ it is easy to see that $z^{\prime} \in_{R}\{0,1\}^{n}$ when $z \in_{R}$ $\{0,1\}^{n}$ and $z=e_{i} \oplus z^{\prime}$. Thus,

$$
\mathrm{E}_{z^{\prime} \in R}\{0,1\}^{n}\left[\overline{B\left(e_{i} \oplus z^{\prime}\right)} \cdot \overline{x \odot z^{\prime}}\right]=\mu_{i} .
$$

The idea is to compute, simultaneously for all $i \in\{1, \ldots, n\}$, a good approximation $Y_{i}$ of $\mu_{i}$. We say that $Y_{i}$ is a good approximation if $\left|Y_{i}-\mu_{i}\right|<\delta$. Define

$$
\operatorname{bit}\left(Y_{i}\right)= \begin{cases}0 & \text { if } Y_{i} \geq 0 \\ 1 & \text { if } Y_{i}<0\end{cases}
$$

Because $\left|\mu_{i}\right| \geq \delta$, if $Y_{i}$ is a good approximation then $\operatorname{bit}\left(Y_{i}\right)=x_{i}$. Let $m=\left[2 n / \delta^{2}\right]$ and let $T_{1}, \ldots, T_{m} \in_{R}\{0,1\}^{n}$ be pairwise independent random variables. Let

$$
Y_{i}=1 / m \cdot \sum_{j=1}^{m} \overline{B\left(e_{i} \oplus T_{j}\right)} \cdot \overline{x \odot T_{j}} .
$$

Then, using the pairwise independence of the random variables and the fact that, for all $j$,

$$
\mathrm{E}\left[\left(\overline{B\left(e_{i} \oplus T_{j}\right)} \cdot \overline{x \odot T_{j}}-\mu_{i}\right)^{2}\right] \leq 1,
$$

it follows that

$$
\mathrm{E}\left[\left(Y_{i}-\mu_{i}\right)^{2}\right] \leq 1 / m .
$$

From Chebychev's inequality it then follows that

$$
\operatorname{Pr}\left[\left|Y_{i}-\mu_{i}\right| \geq \delta\right] \leq \mathrm{E}\left[\left(Y_{i}-\mu_{i}\right)^{2}\right] / \delta^{2} \leq 1 /\left(m \delta^{2}\right) \leq 1 /(2 n) .
$$

From this it follows that

$$
\operatorname{Pr}\left[\exists i \in\{1, \ldots, n\} ;\left|Y_{i}-\mu_{i}\right| \geq \delta\right] \leq 1 / 2,
$$


and so

$$
\operatorname{Pr}\left[\forall i \in\{1, \ldots, n\} ;\left|Y_{i}-\mu_{i}\right|<\delta\right] \geq 1 / 2,
$$

The only remaining difficulty is how to compute $Y_{i}$ given $T_{1}, \ldots, T_{m}$. Everything is relatively easy to compute, except for the values of $x \odot T_{j}$ for all $j \in\{1, \ldots, m\}$. If $T_{1}, \ldots, T_{m}$ are chosen in the obvious way, i.e., each is chosen independently of all the others, then we need to be able to compute $x \odot T_{j}$ correctly for all $j \in\{1, \ldots, m\}$ and there is probably no feasible way to do this. (Recall that we don't know the value of $x$.) Instead, the approach is to take advantage of the fact that the analysis only requires $T_{1}, \ldots, T_{m}$ to be pairwise independent.

Let $\ell=[\log (m+1)]$ and let $v \in\{0,1\}^{n \times \ell}$. Let $T_{1}(v), \ldots, T_{m}(v)$ be as described in the Generalized Inner Product Space (Section 5.2.1), i.e., for all $v \in\{0,1\}^{n \times \ell}$ and for all $j \in\{0,1\}^{\ell}-0^{\ell}, T_{j}(v)=v \odot j$. As we describe, this particular construction allows feasible enumeration of all possible values of $x \odot T_{j}(v)$ for all $j \in\{1, \ldots, m\}$ without knowing $x$. Because of the properties stated above,

$$
x \odot T_{j}(v)=x \odot(v \odot j)=(x \odot v) \odot j .
$$

Thus, it is easy to compute, for all $j \in\{1, \ldots, m\}$, the value of $x \odot T_{j}(v)$ given $x \odot v$. From this we can compute, for all $i \in\{1, \ldots, n\}$,

$$
Y_{i}(v)=1 / m \cdot \sum_{j=1}^{m} \overline{B\left(e_{i} \oplus T_{j}(v)\right)} \cdot \overline{(x \odot v) \odot j} .
$$

The key point is that there are only $2^{\ell}=\mathcal{O}(m)$ possible settings for $x \odot v$, and we try them all. For any $x$ and $v$ there is some $\beta \in\{0,1\}^{\ell}$ such that $\beta=x \odot v$. Let

$$
Y_{i}(\beta, v)=1 / m \cdot \sum_{j=1}^{m} \overline{B\left(e_{i} \oplus T_{j}(v)\right)} \cdot \overline{\beta \odot j},
$$

i.e., $Y_{i}(\beta, v)$ is the value obtained when $\beta$ is substituted for $x \odot v$ in the computation of $Y_{i}(v)$. Consider choosing $v \in_{R}\{0,1\}^{n \times \ell}$. Since from equation (2) above, the probability that $Y_{i}(x \odot v, v)$ is a good approximation for all $i \in\{1, \ldots, n\}$ is at least one-half, it follows that with probability at least one-half there is at least one $\beta \in\{0,1\}^{\ell}$ such that 
$Y_{i}(\beta, v)$ is simultaneously a good approximation for all $i \in\{1, \ldots, n\}$. For this value of $\beta$ and for such a $v,\left\langle\operatorname{bit}\left(Y_{1}(\beta, v)\right), \ldots, \operatorname{bit}\left(Y_{n}(\beta, v)\right)\right\rangle$ is equal to $x$.

\section{Adversary $S^{B}$ on input $\delta>0$ :}

$m \leftarrow\left[2 n / \delta^{2}\right]$,

$\ell \leftarrow[\log (m+1)]$.

$\mathcal{L} \leftarrow \emptyset$.

Choose $v \in_{R}\{0,1\}^{n \times \ell}$.

For all $\beta \in\{0,1\}^{\ell}$ do:

For all $j=1, \ldots, m$ do:

Compute $T_{j}(v)=v \odot j$.

For all $i=1, \ldots, n$ do:

Compute $Y_{i}(\beta, v)=1 / m \cdot \sum_{j=1}^{m} \overline{B\left(e_{i} \oplus T_{j}(v)\right)} \cdot \overline{\beta \odot j}$.

$\mathcal{L} \leftarrow \mathcal{L} \cup\left\{\left\langle\operatorname{bit}\left(Y_{1}(\beta, v)\right), \ldots, \operatorname{bit}\left(Y_{n}(\beta, v)\right)\right\rangle\right\}$.

From the above analysis, it follows that $x \in \mathcal{L}$ with probability at least $1 / 2$, where this probability is over the random choice of $v$.

As long as the running time $T$ for computing $B$ is large compared to $n$ (which it is in our use of the Hidden Bit Technical Theorem to prove the Hidden Bit Theorem), the running time of $S^{B}$ is $\mathcal{O}\left(n^{3} T / \delta^{4}\right)$.

The following exercise shows that the inner product bit is special, i.e., it is certainly not the case that any bit of the input to $f$ is hidden if $f$ is a one-way function.

Exercise 5.6. Describe a one-way permutation $f(x)$ where the first bit of $x$ is not hidden given $f(x)$. Let $f(x)$ be any polynomial-time computable function. Show that if $x_{i}$ can be predicted with probability greater than $1-1 /(2 n)$ given $\langle f(x), i\rangle$ when $x \in_{R}\{0,1\}^{n}$ and $i \in_{R}\{1, \ldots, n\}$ then $f$ is not a one-way function.

The converse of the Hidden Bit Theorem is not true, i.e., there is a function $f$ where the inner product bit is hidden but $f$ is not a one-way function. This is the point of the following exercise. 
Exercise 5.7. Describe a polynomial-time computable function $f(x)$ which is certainly not a one-way function but for which the inner product bit is provably $2^{n}$-secure.

\subsection{Pseudo-random generators}

Blum-Micali [8] introduce the concept of a pseudo-random generator that is useful for cryptographic (and other) applications, and gave it the significance it has today by providing the first provable construction of a pseudo-random generator based on the conjectured difficulty of a wellknown and well-studied computational problem. In particular, both the definition of pseudo-random generator based on the next bit test and the construction of a pseudo-random generator based on the difficulty of the discrete log problem can be found in Blum-Micali [8].

Yao [41] introduces the definition (below) of a pseudo-random generator, and shows an equivalence between this definition and the next bit test introduced in Blum-Micali [8]. The standard definition of a pseudorandom generator of Yao is based on the concept of computational indistinguishability introduced previously in Goldwasser-Micali [16].

Definition 5.8. (pseudo-random generator) Let $g(x)$ be a polynomial-time computable function where $\ell(n)=\|g(x)\|, n=\|x\|$, and $\ell(n)>n$. The stretching parameter of $g(x)$ is $\ell(n)-n$. The success probability (distinguishing probability) of adversary $A$ for $g$ is

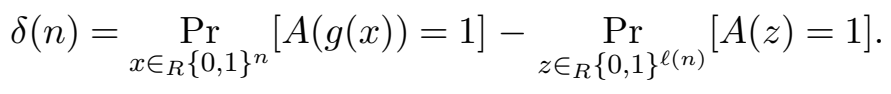

Then, $g$ is a $\mathbf{S}(n)$-secure pseudo-random generator if every $A$ has time/success ratio at least $\mathbf{S}(n)$.

The following exercise shows that an immediate application of the Hidden Bit Theorem is the construction of a pseudo-random generator from a one-way permutation.

Exercise 5.9. From the Hidden Bit Theorem, show that if $f(x)$ is a one-way permutation then $g(x, z)=\langle f(x), z, x \odot z\rangle$ is a pseudo-random 
generator that stretches by 1 bit. The reduction should describe a TM $M$ with the property that if $A$ is an adversary for distinguishing $g$ with time/success ratio $\mathbf{S}(n)$ then $M^{A}$ is an adversary for inverting $f$ with time/success ratio $\mathbf{S}(n)^{c}$ for some constant $c>0$.

The simple construction of a pseudo-random generator given in the previous exercise was one of the motivating forces behind the work of Goldreich-Levin [15]. The reduction from an arbitrary one-way function to a pseudo-random generator can be found in Håstad-ImpagliazzoLevin-Luby [19].

We can construct a pseudo-random generator that stretches by an arbitrary polynomial amount based on any one-way permutation. Let $f(x)$ be a one-way permutation. Define $g(x, z)$ where $\|z\|=\|x\|=n$, as

$$
g(x, z)=\left\langle z, x \odot z, f(x) \odot z, f^{(2)}(x) \odot z, \ldots, f^{(\ell(n)-n-1)}(x) \odot z\right\rangle,
$$

where $f^{(i)}$ is the function $f$ composed with itself $i$ times.

Theorem 5.10. If $f$ is a one-way permutation then $g$ is a pseudorandom generator. In particular, there is a TM $M$ with the property that if $A$ is an adversary for distinguishing $g$ with time/success ratio $\mathbf{S}(n)$ then $M^{A}$ is an adversary for inverting $f$ with time/success ratio $\mathbf{S}(n)^{c}$ for some constant $c>0$.

This theorem is a combination of a theorem due to GoldreichGoldwasser-Micali [14] and the Hidden Bit Theorem (see Section 5.2) of Goldreich-Levin [15].

Exercise 5.11. Prove the above theorem. 



\section{6}

\section{Deterministic Counting}

In this chapter we consider the complexity of several "approximate counting" problems. Informally this is the class of prolems derived from NP languages, where the goal now is to count the number of witnesses that prove membership of a given input in the language.

\section{1 \#P and approximate counting}

Recall that a language $\mathcal{L} \in \mathbf{N P}$ if there is an associated TM $M$ such that, for all $x \in\{0,1\}^{n}, x \in \mathcal{L}$ iff $\left|W_{x}\right| \geq 1$, where $W_{x}=\mid\left\{y \in\{0,1\}^{r}\right.$ : $M(x, y)=1\} \mid$. A function $f \in \# \mathbf{P}$ if there is an NP language $\mathcal{L}$ with an associated TM $M$ such that, for all $x \in\{0,1\}^{n}, f(x)=\left|W_{x}\right|$. In words, $f(x)$ is the number of witnesses that show $x \in \mathcal{L}$. In particular, note that $f(x)=0$ iff $x \notin \mathcal{L}$, and thus it is clear that a polynomial time algorithm for computing $f$ immediately implies $\mathbf{P}=\mathbf{N P}$. The definition of the complexity class $\# \mathbf{P}$, and the realization of its importance, are due to Valiant [38]. Examples of $f \in \# \mathbf{P}$ are the following:

- If $x$ is the description of a graph then $f(x)$ is the number of perfect matchings in the graph, else $f(x)=0$.

- If $x$ is the description of a graph then $f(x)$ is the number of Hamiltonian tours in the graph, else $f(x)=0$. 
- If $x$ is the description of a DNF boolean formula then $f(x)$ is the number of truth assignments that satisfy the formula, else $f(x)=0$.

- If $x$ is the description of a CNF boolean formula then $f(x)$ is the number of truth assignments that satisfy the formula, else $f(x)=0$.

As there is a notion of completeness for NP, there is an analogous notion of completeness for $\# \mathbf{P}$. Intuitively, if a function $f$ is \#P-complete and if there is a polynomial time algorithm for computing $f$ then there is a polynomial time algorithm for computing every $g \in \# \mathbf{P}$.

As shown in Valiant [38], all four examples described above are \#P-complete functions. Most often it is the case that if the language $\mathcal{L}$ is $\mathbf{N P}$-complete then it takes little effort to show that the associated counting problem $f$ is $\# \mathbf{P}$-complete, and this is the case for second and fourth examples. The first and third examples are more interesting because the associated NP-language can be decided in polynomial time. The proof that the third example is $\# \mathbf{P}$-complete is rather

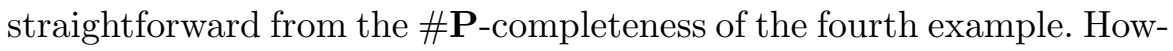
ever, the $\# \mathbf{P}$-completeness of the first example is not at all straightforward.

It turns out that many important counting problems are \# $\mathbf{P}$-complete, and unless $\mathbf{P}=\mathbf{N P}$ there is no hope of finding polynomial time algorithms for these problems. On the other hand, in practice it is often useful to provide a good approximation of the number of solutions. As before, let $\mu\left(W_{x}\right)=\left|W_{x}\right| / 2^{r}$ be the fraction of witnesses for $x$ among all possible witnesses. There are two potential definitions of what a good estimate means:

(1) $Y$ is an $\epsilon$-good absolute approximation of $\mu\left(W_{x}\right)$ if

$$
\mu\left(W_{x}\right)-\epsilon \leq Y \leq \mu\left(W_{x}\right)+\epsilon .
$$

(2) $Y$ is an $\epsilon$-good relative approximation of $\mu\left(W_{x}\right)$ if

$$
\mu\left(W_{x}\right)(1-\epsilon) \leq Y \leq \mu\left(W_{x}\right)(1+\epsilon) .
$$


An estimate $Y$ is more useful and meaningful if it is an $\epsilon$-good relative approximation, especially in the typical case when $\mu\left(W_{x}\right)$ is small.

In recent years, a body of work has been devoted to finding fast algorithms to approximate $\# \mathbf{P}$-complete functions. Let $f$ be a $\# \mathbf{P}$ function. Following Karp-Luby [23], we say a randomized algorithm $A$ provides a fully polynomial randomized approximation scheme (abbreviated to fpras) for $f$ if, for every pair of positive input parameters $(\epsilon, \delta)$ and for every input $x \in\{0,1\}^{n}$,

(1) $A(x, \epsilon, \delta)$ is an $\epsilon$-good relative approximation of $f(x)$ with probability at least $1-\delta$. The probability is with respect to the source of randomness used by $A$.

(2) The run time $A(x, \epsilon, \delta)$ is bounded by a polynomial in $\|x\|$, $1 / \epsilon$ and $\log (1 / \delta)$.

Let us say that $A$ is a weak fpras if requirement (1) in the above definition is changed to say that $A(x)$ is an $\epsilon$-good absolute approximation instead of an $\epsilon$-good relative approximation. Based on the standard sampling algorithm described below, it is easy to see there is a weak fpras for every $f \in \# \mathbf{P}$. On the other hand, a fpras for the CNF counting problem immediately implies $\mathbf{R P}=\mathbf{N P}$.

In the following sections, we develop a fpras for the DNF counting problem and for a related problem. The following simple and standard sampling algorithm at a very high level provides the general outline for the algorithms for both problems. Suppose we have a finite (but large) universe $U$ of known size $|U|$, and our goal is to estimate the size of some set $G \subset U$ of unknown size. A trial of the algorithm for estimating $|G|$ consists of the following two steps:

(1) Choose $s \in_{R} U$.

(2) See if $s \in G$.

Let $b$ be an easily computable upper bound on $|U| /|G|$. The algorithm performs $N=4 b \ln (2 / \delta) / \epsilon^{2}$ independent trials, and the output $Y$ is the fraction of these $N$ trials where an element of $G$ is chosen, multiplied by $|U|$. A standard analysis using an inequality due to Bernstein 
Renyi [35] shows that for $\epsilon<1$,

$$
\operatorname{Pr}[|G|(1-\epsilon) \leq Y \leq|G|(1+\epsilon)] \geq 1-\delta .
$$

(See for example Karp-Luby-Madras [24] for a proof.)

The key points about the sampling algorithm are the following:

(a) The universe $U$ should be defined in such a way that $|U|$ is easy to compute.

(b) Steps (1) and (2) of the trial can be performed efficiently.

(c) $|G|$ is known a priori to be a significant fraction of $|U|$, i.e. the upper bound $b$ on their ratio is polynomially bounded, and computable in polynomial time.

\subsection{DNF counting}

Let $y=\left\langle y_{1}, \ldots, y_{r}\right\rangle$ be a collection of $r$-boolean variables and let $F$ be a boolean formula in disjunctive normal form (DNF formula); i.e, $F=$ $c_{1} \vee c_{2} \vee \cdots \vee c_{m}$, where $c_{i}=z_{i_{1}} \wedge \cdots \wedge z_{i_{\ell_{i}}}$, for some set of literals

$$
\left\{z_{i_{1}}, \ldots, z_{i_{\ell_{i}}}\right\} \subseteq\left\{y_{1}, \ldots, y_{r}, \bar{y}_{1}, \ldots, \bar{y}_{r}\right\} .
$$

For a truth assignment $a \in\{0,1\}^{r}$ to $y$, let $M(F, a)=1$ if $a$ satisfies $F$, and let $M(F, a)=0$ otherwise. Let $f(F)=\left|\left\{a \in\{0,1\}^{r}: M(F, a)=1\right\}\right|$. It is clear that $f \in \# \mathbf{P}$, and, as mentioned before, $f$ is $\# \mathbf{P}$-complete. We describe below a fpras algorithm for $f$ due to Karp-Luby [23], KarpLuby-Madras [24].

A naive approach to approximate $f(F)$ is the following. Let the sample space be the set $\{0,1\}^{r}$ of all possible truth assignments. Choose several random truth assignments, and estimate $f(F)$ by the fraction of these truth assignments that satisfy $F$. The problem with this approach is that if $F$ is satisfied by an exponentially small percentage of truth assignments then this approach requires an exponential number of samples.

Instead, we design the following sample space. Let $C_{i}$ be the set of truth assignments that satisfy clause $c_{i}$. Let

$$
U=\left\{(i, a): i \in\{1, \ldots, m\} \wedge a \in C_{i}\right\}
$$


and let $G \subseteq U$ be defined by

$$
G=\{(i, a) \in U: \text { there is no } j<i \text { such that }(j, a) \in U\} .
$$

Notice that $|U|=\sum_{i \in\{1, \ldots, m\}}\left|C_{i}\right|$, and $\left|C_{i}\right|=2^{r-\ell_{i}}$, and thus $|U|$ is easy to compute. Note also that $|G|=f(F)$, because for each truth assignment $a$ that satisfies $F$ there is a unique smallest index $i$ such that $a \in C_{i}$. Furthermore,

$$
\frac{|U|}{|G|} \leq \frac{\sum_{i \in\{1, \ldots, m\}}\left|C_{i}\right|}{\max _{i \in\{1, \ldots, m\}}\left|C_{i}\right|} \leq m .
$$

Therefore, from Equation 6.1 of the previous section, we can approximate $f(F)$ with $N=\frac{4 m}{\epsilon^{2}} \log \left(\frac{1}{\delta}\right)$ trials. In each trial we will

1. Choose index $i \in\{1, \ldots, m\}$ with probability $\left|C_{i}\right| /|U|$. This requires time $\mathcal{O}(\log m)$ time (with some preprocessing, we leave as an easy exercise what preprocessing to do to achieve this time bound.)

2. Choose $a \in_{R} C_{i}$. This step takes $\mathcal{O}(r)$ time.

3. See if $(i, a) \in G$. This can be done in the obvious way in time $\mathcal{O}(\mathrm{rm})$.

4. The value produced by the trial is $|U|$ if $(i, a) \in G$, and 0 otherwise.

The overall estimate is the average of the values produced by the $N$ trials. By Equation 6.1 this is guaranteed to be an $\epsilon$-good relative estimate of $f(F)$ with probability at least $1-\delta$. The overall running time is $\mathcal{O}\left(\frac{r m^{2}}{\epsilon^{2}} \log \left(\frac{1}{\delta}\right)\right)$.

\subsection{GF[2] polynomial counting}

Let $y=\left\langle y_{1}, \ldots, y_{r}\right\rangle$ be a collection of $r$ variables over $\mathrm{GF}[2]$ and let $F$ be a polynomial over $\mathrm{GF}[2]$ with respect to the variables $y$ i.e., $F=t_{1} \oplus t_{2} \oplus \cdots \oplus t_{m}$, where term $t_{i}=y_{i_{1}} \odot \cdots \odot y_{i_{\ell_{i}}}$ for some subset $\left\{y_{i_{1}}, \ldots, y_{i_{\ell_{i}}}\right\}$ of $y$. For an assignment $a \in\{0,1\}^{r}$ to $y$, let $M(F, a)=1$ if $a$ is a zero of $F$, i.e., $a$ satisfies an even number of the $m$ terms of $F$, 
and $M(F, a)=0$ otherwise. Let $f(F)=\left|\left\{a \in\{0,1\}^{r}: M(F, a)=1\right\}\right|$. It is \# $\mathbf{P}$-complete to compute $f$. The following fpras for approximating $f$ is due to Karpinski-Luby [26].

We design two different fpras algorithms $A_{0}$ and $A_{1} ; A_{0}$ is used in the case when $F$ does not contain the constant term 1 and $A_{1}$ is used in the case when $F$ contains the term 1 . Note that the term 1 corresponds to the product of the empty set of variables, and is satisfied by all assignments to $y$. The analyses of the two algorithms are very similar. The running time of $A_{0}$ is $\mathcal{O}\left(\mathrm{rm}^{2} \ln (1 / \delta) / \epsilon^{2}\right)$ and the running time of $A_{1}$ is $\mathcal{O}\left(\mathrm{rm}^{3} \ln (1 / \delta) / \epsilon^{2}\right)$.

We first describe algorithm $A_{0}$. Let $U$ be the set $\{0,1\}^{r}$ of all assignments to $y$, and let $H_{\text {even }}$ be the set of all assignments that satisfy an even number of terms. A trial of the algorithm consists of choosing an assignment $a \in_{R}\{0,1\}^{r}$ and testing if $a \in H_{\text {even }}$. The outcome of a trial is $|U|=2^{r}$ if $a \in H_{\text {even }}$ and the outcome is 0 otherwise. The output of the algorithm is the average of the outcomes of all the trials.

The most time consuming part of the trial is to test if $a \in H_{\text {even }}$, and this takes time $\mathcal{O}(\mathrm{rm})$. The corollary at the end of this section shows that $|U| /\left|H_{\text {even }}\right| \leq m+1$, and thus $N=4(m+1) \ln (2 / \delta) / \epsilon^{2}$ trials suffice. Thus, the total running time of $A_{0}$ is $\mathcal{O}\left(r m^{2} \ln (2 / \delta) / \epsilon^{2}\right)$.

We now describe algorithm $A_{1}$. The outline of the algorithm is borrowed from the DNF approximation algorithm described in the previous section. We provide a self-contained description of the algorithm. Let $F$ be the input polynomial with the constant term 1 discarded. Thus, the problem is to approximate the number of assignments that satisfy an odd number of terms of $F$. For all $i=1, \ldots, m$, let $T_{i}$ be the set of assignments that make term $t_{i}$ evaluate to 1 . Analogous to the DNF algorithm, let

$$
U=\left\{(i, a): i \in\{1, \ldots, m\} \wedge a \in T_{i}\right\}
$$

and let $G \subseteq U$ be defined by

$$
G=\{(i, a) \in U: \text { there is no } j<i \text { such that }(j, a) \in U\} .
$$

Let $G_{\text {odd }} \subseteq G$ be defined by

$$
G_{\text {odd }}=\left\{(i, a) \in G:\left|\left\{j \in\{1, \ldots, m\}: j \in T_{i}\right\}\right| \bmod 2=1\right\},
$$


i.e., $(i, a)$ is in $G_{\text {odd }}$ if it is in $G$ and if $a$ makes an odd number of terms evaluate to 1 . The key point is that $\left|G_{\text {odd }}\right|$ is the quantity we want to approximate. One trial of the algorithm consists of choosing $(i, a) \in_{R} U$ and then testing if $(i, a) \in G_{\text {odd }}$ : if yes then the value produced by the trial is $|U|$, else the value is 0 .

We now verify that the efficiency criteria (a), (b) and (c) described at teh end of Section 6.1 are satisfied. The computation of $|U|$ and the method for choosing $(i, a) \in_{R} U$ is analogous to the method for the DNF approximation algorithm described in the previous section. The most time consuming part of the trial is to test if $(i, a) \in G_{\text {odd }}$, and this takes $\mathcal{O}(r m)$ time.

The final portion of the analysis is to show that $|U| /\left|G_{\text {odd }}\right|$ is not too large. As described in the previous section, $|U| /|G| \leq m$. The theorem given below shows that $|G| /\left|G_{\text {odd }}\right| \leq m$. It follows that $|U| /\left|G_{\text {odd }}\right| \leq m^{2}$, and thus from Equation 6.1 it follows that $N=$ $4 m^{3} \ln (2 / \delta) / \epsilon^{2}$ trials suffice. Thus, the total running time of $A_{1}$ is $\mathcal{O}\left(r m^{3} \ln (2 / \delta) / \epsilon^{2}\right)$.

Theorem 6.1. Let $F$ be a multivariate polynomial over GF[2] with no duplicate terms and $m$ terms in total. Let $H$ be the set of assignments to the variables that satisfy at least one term, and let $H_{\text {odd }}$ be the set of assignments that satisfy an odd number of terms. Then,

$$
|H| /\left|H_{\text {odd }}\right| \leq m \text {. }
$$

Proof. The basic idea of the proof is to define a function $h: H \rightarrow H_{\text {odd }}$ in such a way that the mapping is at most $m$-to- 1 , i.e. for each $a \in$ $H_{\text {odd }},\left|h^{-1}(a)\right| \leq m$. From this the theorem follows.

The mapping $h$ is defined as follows. For each $a \in H$, choose any term $t_{i}$ that is satisfied by $a$ such that there is no term $t_{j}$ which is satisfied and which contains all the variables in $t_{i}$. It is always possible to choose such a term because $F$ does not contain two identical terms. Without loss of generality, let this be term $t_{1}$ and let $S=\left\{y_{1}, \ldots, y_{k}\right\}$ be the variables in $t_{1}$ (all of these variables are equal to 1 in $a$ ). For any $S^{\prime} \subseteq S$, let $a\left(S^{\prime}\right)$ be the assignment obtained from $a$ by changing the values of all variables in $S-S^{\prime}$ from 1 to 0 . 
Claim 6.2. There is at least one $S^{\prime} \subseteq S$ such that $a\left(S^{\prime}\right)$ satisfies an odd number of terms of $F$.

Proof of Claim: For each $S^{\prime} \subseteq S$, let $p\left(S^{\prime}\right)$ be the parity of the number of terms that are satisfied by assignment $a\left(S^{\prime}\right)$ and let $q\left(S^{\prime}\right)$ be the parity of the number of terms $t_{i}$ such that $t_{i} \cap S=S^{\prime}$. By the way term $t_{1}$ is chosen, $t_{1}$ is the only term $t_{i}$ that satisfies $t_{i} \cap S=S$, and thus $q(S)=1$. We can view $p(\cdot)$ and $q(\cdot)$ as column vectors of length $2^{k}$ with entries from GF[2], where the first entry corresponds to $S^{\prime}=\emptyset$ and the last entry corresponds to $S^{\prime}=S$. Then it can be verified that there is a $2^{k} \times 2^{k}$ lower triangular matrix $R$ over GF[2] with main diagonal 1 such that $R \odot q(\cdot)=p(\cdot)$. In particular, row $S^{\prime}$ in $R$ has a 1 in column $S^{\prime \prime}$ if and only if $S^{\prime \prime} \subseteq S^{\prime}$. (See Figure 6.1.)

Because $R$ is invertible over GF[2] and because $q(\cdot) \not \equiv 0$, it follows that for at least one $S^{\prime} \subseteq S, p\left(S^{\prime}\right)=1$. For this $S^{\prime}, a\left(S^{\prime}\right)$ satisfies an odd number of terms. This complete the proof of the claim.

We now complete the proof of the theorem. To define $h(a)$, we arbitrarily choose any $S^{\prime}$ such that $a\left(S^{\prime}\right)$ satisfies an odd number of terms and let $h(a)=a\left(S^{\prime}\right)$. Finally, we argue that for each $b \in H_{\text {odd }}$ there are at most $m$ distinct assignments $a \in H$ such that $h(a)=b$. This is because each such $a$ is either equal to $b$, or is obtained by

\begin{tabular}{c|cccccccc} 
& $\emptyset$ & $\{1\}$ & $\{2\}$ & $\{3\}$ & $\{1,2\}$ & $\{1,3\}$ & $\{2,3\}$ & $\{1,2,3\}$ \\
\hline$\emptyset$ & 1 & 0 & 0 & 0 & 0 & 0 & 0 & 0 \\
$\{1\}$ & 1 & 1 & 0 & 0 & 0 & 0 & 0 & 0 \\
$\{2\}$ & 1 & 0 & 1 & 0 & 0 & 0 & 0 & 0 \\
$\{3\}$ & 1 & 0 & 0 & 1 & 0 & 0 & 0 & 0 \\
$\{1,2\}$ & 1 & 1 & 1 & 0 & 1 & 0 & 0 & 0 \\
$\{1,3\}$ & 1 & 1 & 0 & 1 & 0 & 1 & 0 & 0 \\
$\{2,3\}$ & 1 & 0 & 1 & 1 & 0 & 0 & 1 & 0 \\
$\{1,2,3\}$ & 1 & 1 & 1 & 1 & 1 & 1 & 1 & 1
\end{tabular}

Fig. 6.1 The matrix $R$ for $k=3$ 
taking one of the terms not satisfied by $b$ (there are at most $m-1$, since $b$ must satisfy an odd number and thus at least one term) and setting the values of all variables in this term to 1 .

Note that the theorem holds even in the case when $F$ contains the constant term 1 . This fact is used in the proof of the following corollary.

Corollary 6.3. Let $F$ be a multivariate polynomial over GF[2] with no duplicate terms, no occurence of the constant term 1 and $m$ terms in total. Let $U$ be the set of all assignments to the variables, and let $H_{\text {even }}$ be the set of assignments that satisfy an even number of terms. Then, $|U| /\left|H_{\text {even }}\right| \leq m+1$.

Proof. Let $\bar{F}=F \oplus 1$. Then, $\bar{H}_{\text {odd }}=H_{\text {even }}$. Because $F$ does not contain the term $1, \bar{F}$ contains $m+1$ terms in total, no duplicate terms, and every assignment satisfies the constant 1 term, and thus $\bar{H}=U$. By the above theorem, $|U| /\left|H_{\text {even }}\right|=|\bar{H}| /\left|\bar{H}_{\text {odd }}\right| \leq m+1$.

The bound given in the theorem is optimal. To see this, let $m$ be a power of two and let $F=\prod_{i=1, \ldots, \log m}\left(1 \oplus y_{i}\right) \prod_{j=\log m, \ldots, r} y_{j}$. When $F$ is viewed as a polynomial over GF[2] and expanded out the number of terms is $m$. When viewed over GF[2], $F=1$ has a unique solution, whereas when viewed as a DNF formula, $F$ has $m$ satisfying assignments.

The bound given in the above corollary is also optimal. To see this, consider $F=1 \oplus \prod_{i=1, \ldots, r}\left(1 \oplus y_{i}\right)$. When $F$ is viewed as a polynomial over GF[2] and expanded out the 1 term is cancelled and the total number of terms is $m=2^{r}-1$. When viewed over GF[2], $F=0$ has a unique solution, and thus $|U| /\left|H_{\text {even }}\right|=2^{r}=m+1$.

\subsection{Bounded depth circuit counting}

Recall that in Sections 4.1-4.7 we introduced ways to reduce the amount of randomness when amplifying the probability of correctly deciding membership of $x$ with respect to an $\mathbf{R P}$ or BPP language $\mathcal{L}$. However, the number of random bits needed was still at least the 
number to choose a single potential witness at random, i.e., at least $r$ bits. In this section, we show how to deterministically decide membership for a BPP language $\mathcal{L}$ where the NP TM $M$ associated with $\mathcal{L}$ is restricted to be expressible as a constant depth unbounded fanin circuit. While this restriction on $M$ may seem to be severe at first glance (and it is), such a machine $M$ is nevertheless powerful enough to express a rich class of \#P-complete problems. For example, for the DNF counting problem described in Section 6.2, the associated TMM can be expressed as a depth 2 unbounded fan-in circuit.

Definition 6.4. $\left(\mathcal{C}_{n}^{d}\right)$ Let $\mathcal{C}_{n}^{d}$ be the set of all circuits with $n$ boolean input variables $z=\left\langle z_{1}, \ldots, z_{n}\right\rangle$ of depth $d$. A circuit $C \in \mathcal{C}_{n}^{d}$ consists of $\wedge$-gates and $\vee$-gates, where each gate is allowed unbounded fan-in. $C$ consists of $d$ levels of gates, where all gates at a given level are the same type. All the gates at level 1 have as inputs any mixture of variables and their negations. For all $i \in\{2, \ldots, d\}$, all gates at level $i$ receive their inputs from the gates at level $i-1$. There is a single gate at level $d$, and either its value or the negation of its value is considered to be the output $C(z) \in\{0,1\}$ of $C$.

For $x \in\{0,1\}^{n}, x \odot x=\oplus_{i \in\{1, \ldots, n\}} x_{i}$ is the parity of the number of ones in $x$.

Definition 6.5. (predicting the parity of its inputs) For any circuit $C \in \mathcal{C}_{n}^{d}$, let $p_{C}$ be the prediction probability of $C$ for the parity of its input, i.e.,

$$
p_{C}=\operatorname{Pr}_{z \in_{R}\{0,1\}^{n}}[C(z)=z \odot z]-1 / 2 .
$$

Let $T_{C}$ be the total number of gates in $C$. The time/success ratio of $C$ for predicting the parity of its inputs is $S_{C}=T_{C} / p_{C}$.

The following lower bound theorem is a culmination of a number of papers, i.e., Furst-Saxe-Sipser [13], Ajtai [2], Yao[42], Cai[9], Håstad[18].

Parity Theorem: There is a constant $\kappa>0$ such that for any $C \in \mathcal{C}_{n}^{d}$, the time/success ratio $S_{C}$ of $C$ satisfies $S_{C} \geq 2^{n^{\kappa / d}}$. 
Let $\mathcal{L} \in \mathbf{B P P}$ and let $M$ be the $\mathbf{T M}$ associated with $\mathcal{L}$ with associated constants $c_{\text {yes }}$ and $c_{\text {no }}$. Suppose that the computation of $M(x, y)$ for a fixed value of $x \in\{0,1\}^{n}$ as a function of $y \in\{0,1\}^{r}$ can be expressed as a circuit $C \in \mathcal{C}_{r}^{d}$. Let $g:\{0,1\}^{\ell} \rightarrow\{0,1\}^{r}$ be a function.

Definition 6.6. (distinguishing probability of $C$ for $g$ ) For a circuit $C \in \mathcal{C}_{r}^{d}$, we let $\delta_{C}$ be the distinguishing probability of $C$ for $g$ (analogous to the definition of a pseudo-random generator in Section 5.3), i.e.,

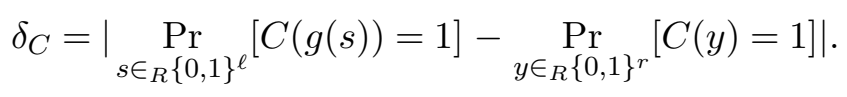

Let $T_{C}$ be the total number of gates in $C$. The time/success ratio of $C$ for distinguishing $g$ is $S_{C}=T_{C} / \delta_{C}$.

The key to the constructions of Nisan[30], Nisan-Wigderson [32] is to design $g$ in such a way that the following properties hold:

- The length $\ell$ of the input to $g$ is much shorter than the length $r$ of its output.

- The time to compute $g(s) \in\{0,1\}^{r}$ given $s \in\{0,1\}^{\ell}$ is polynomial in $r$.

- The distinguishing probability $\delta_{C}$ of $C$ for $g$ satisfies

$$
\delta_{C}<\left(c_{\text {yes }}-c_{\text {no }}\right) / 2
$$

Given these properties, to decide membership of $x \in \mathcal{L}$ is easy: Simply run $C(g(s))$ for all $s \in\{0,1\}^{\ell}$, and then decide $x \in \mathcal{L}$ iff the fraction of these inputs on which $C$ produces the value 1 is at least $\left(c_{\text {yes }}+c_{\text {no }}\right) / 2$. It is not hard to verify that membership of $x$ in $\mathcal{L}$ is always decided correctly. The run time for this procedure is $2^{\ell}$ times the time for computing $g$ on inputs of length $\ell$ (in the construction below, this takes time that is almost linear in the length $r$ of the output of $g$ ) plus the time for computing $C$ on inputs of length $r$.

Note that we can view $g$ as generating a distribution on the $r$-bit input to $C$ consisting of only $2^{\ell}$ sample points that appears pseudorandom to $C$. If it were possible to set $\ell=c \log (r)$ for some constant 
$c>0$ then membership of $x$ in $\mathcal{L}$ could be decided in polynomial time. If this value of $\ell$ were achievable for $C \in \mathcal{C}_{r}^{r}$ (namely, no depth restriction) then this would imply that $\mathbf{B P P}=\mathbf{P}$. (Contrast this with the results of Exercise 4.2.)

We now describe the generator $g$ of Nisan [30], NisanWigderson [32]. Set

$$
\ell=\log (r)^{c(d+1)},
$$

where $c>0$ is a parameter that can be thought of as constant. Let $t_{1}, \ldots, t_{r} \subset\{1, \ldots, \ell\}$ be sets that satisfy the following two properties:

(1) For all $i \in\{1, \ldots, r\},\left|t_{i}\right|=\sqrt{\ell}$.

(2) For all $i, j \in\{1, \ldots, r\}, i \neq j,\left|t_{i} \cap t_{j}\right| \leq \log (r)$.

We leave as Exercise 6.9 the efficient construction of the sets $t_{1}, \ldots, t_{r}$ with these two properties. For all $s \in\{0,1\}^{\ell}$, for each $i \in$ $\{1, \ldots, r\}$, define function $b_{i}(s)=\oplus_{j \in t_{i}} s_{j}$, i.e., $b_{i}(s)$ is the parity of the number of ones in the bits of $s$ indexed by $t_{i}$. Finally, let

$$
g(s)=\left\langle b_{1}(s), \ldots, b_{r}(s)\right\rangle .
$$

The following theorem is due to Nisan [31], Nisan-Wigderson [32].

Theorem 6.7. Let $q(r)=2^{\log (r)^{c \kappa / 2}} / r^{3}$. For all $C \in \mathcal{C}_{r}^{d}$, the time/ success ratio $S_{C}$ of $C$ for distinguishing $g$ satisfies $S_{C} \geq q(r)$.

Proof. Suppose that $C \in \mathcal{C}_{r}^{d}$ has size $T_{C}$ and distinguishing probability $\delta_{C}$ that satisfies $T_{C} / \delta_{C}<q(r)$. We show this implies there is a circuit $C^{\prime} \in \mathcal{C}_{\sqrt{\ell}}^{d+1}$ such that the size $T_{C^{\prime}}$ of $C^{\prime}$ is at most $r^{2}+T_{C}$ and such that the prediction probability $p_{C^{\prime}}$ of $C^{\prime}$ for the parity of its inputs is at least $\delta_{C} / r$. From this it follows that the time/success ratio

$$
T_{C^{\prime}} / p_{C^{\prime}} \leq r^{3} \cdot T_{C} / \delta_{C}<2^{(\sqrt{\ell})^{\frac{\kappa}{d+1}}},
$$

and by the Parity Theorem, such a circuit $C^{\prime}$ with $\sqrt{\ell}$ inputs of depth $d+1$ cannot exist. Thus, it must be the case that $S_{C} \geq q(r)$.

The circuit $C^{\prime}$ will be derived from $C$ and $g$ based on the properties of the generator $g$. We first use a hybrid argument first used by Yao [41] 
that has become standard. Let $s \in_{R}\{0,1\}^{\ell}$ and $y \in_{R}\{0,1\}^{r}$. Consider the following sequence of $r+1$ distributions on $r$-bit strings:

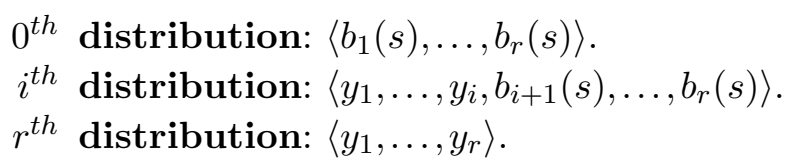

Let $R_{i} \in\{0,1\}^{r}$ be the random variable distributed according to the $i^{\text {th }}$ distribution, and let $p_{i}=\operatorname{Pr}_{R_{i}}\left[C\left(R_{i}\right)=1\right]$. Note that $R_{0}=g(s)$ and $R_{r}=y$, and thus

$$
\delta_{C}=\left|\operatorname{Pr}_{R_{0}}\left[C\left(R_{0}\right)=1\right]-\underset{R_{r}}{\operatorname{Pr}}\left[C\left(R_{r}\right)=1\right]\right|=\left|p_{0}-p_{r}\right| .
$$

Assume without loss of generality that $p_{0}-p_{r}$ is positive. It follows from the triangle inequality that there is some $i \in\{1, \ldots, r\}$ such that

$$
\delta_{i}=p_{i-1}-p_{i} \geq \delta_{C} / r
$$

Fix such an $i$. Note that $R_{i-1}$ and $R_{i}$ both depend only on $s$ and on $y_{1}, \ldots, y_{i}$, and that the only difference between $R_{i-1}$ and $R_{i}$ is that the $i^{\text {th }}$ bit of $R_{i-1}$ is $b_{i}(s)$ and the $i^{\text {th }}$ bit of $R_{i}$ is $y_{i}$.

Without loss of generality, let $t_{i}=\{1, \ldots, \sqrt{\ell}\}$, i.e., $b_{i}(s)$ depends on the first $\sqrt{\ell}$ bits of $s$. Let $s^{\prime}=\left\langle s_{1}, \ldots, s_{\sqrt{\ell}}\right\rangle$. By an averaging argument, there is a setting of values for $y_{1}, \ldots, y_{i}$ and $s_{\sqrt{\ell}+1}, \ldots, s_{\ell}$ such that the distinguishing probability of $C$ for $R_{i-1}$ and $R_{i}$ remains at least $\delta_{i}$ conditional on these fixed values for $y_{1}, \ldots, y_{i}$ and $s_{\sqrt{\ell}+1}, \ldots, s_{\ell}$. Note that in the conditional distributions both $R_{i-1}$ and $R_{i}$ both depend only on $s^{\prime}$.

Let $F^{\prime}\left(s^{\prime}, a\right)$ be the function of $s^{\prime}$ which can be thought of as computing $C$ where the first $i-1$ inputs bits are set to the values for $y_{1}, \ldots, y_{i-1}$ fixed above, the $i^{\text {th }}$ input is set to $a \in\{0,1\}$, and the remaining $r-i$ input bits are computed as $b_{i+1}^{\prime}\left(s^{\prime}\right), \ldots, b_{r}^{\prime}\left(s^{\prime}\right)$, where $b_{j}^{\prime}\left(s^{\prime}\right)$ is the value of $b_{j}\left(s^{\prime}, s_{\sqrt{\ell}+1}, \ldots, s_{\ell}\right)$ when $s_{\sqrt{\ell}+1}, \ldots, s_{\ell}$ are fixed as described above. The above analysis shows that

$$
\delta_{i} \leq \operatorname{Pr}_{s^{\prime} \in_{R}\{0,1\} \sqrt{\ell}}\left[F^{\prime}\left(s^{\prime}, s^{\prime} \odot s^{\prime}\right)=1\right]-\operatorname{Pr}_{s^{\prime} \in_{R}\{0,1\}^{\sqrt{\ell}}}\left[F^{\prime}\left(s^{\prime}, y_{i}\right)=1\right] .
$$

There are two cases to consider, i.e., when $y_{i}=1$ or $y_{i}=0$. We assume that $y_{i}=1$, as the case when $y_{i}=0$ is similar. We leave it as an 
exercise to prove that in this case the prediction probability $p_{F^{\prime}}$ of $F^{\prime}\left(s^{\prime}, 1\right)$ for the parity of its inputs is at least $\delta_{i} \geq \delta_{C} / r$. The intuition is that $F^{\prime}\left(s^{\prime}, a\right)$ is more biased towards producing a 1 when $a=s^{\prime} \odot s^{\prime}$ then when $a=1$, and thus if $F^{\prime}\left(s^{\prime}, 1\right)$ produces a 1 it is more likely that $s^{\prime} \odot s^{\prime}=1$ then 0 , whereas if $F^{\prime}\left(s^{\prime}, 1\right)=0$ then it is more likely that $s^{\prime} \odot s^{\prime}=0$ then 1 .

We now show that $F^{\prime}\left(s^{\prime}, y_{i}\right)$ can be computed by a small circuit $C^{\prime}\left(s^{\prime}\right)$ of depth $d+1$. Note that since for all $j \in\{i+1, \ldots, r\}, \mid t_{j} \cap$ $t_{i} \mid \leq \log (r)$, it follows that $b_{j}^{\prime}\left(s^{\prime}\right)$ depends on at most $\log (r)$ bits of $s^{\prime}$. Furthermore, any function of $\kappa$-bits can be expressed as either a DNF or CNF circuit with at most $2^{k}$ gates at the first level. Suppose without loss of generality that the first level of gates of $C$ are $\wedge$-gates. Then, we can express $b_{j}^{\prime}\left(s^{\prime}\right)$ as a DNF circuit $C_{j}$ with at most $r \vee$-gates at the first level and a single $\wedge$-gate at the second level. We can then merge $C_{j}$ into the circuit $C$ by feeding all the values of the $\vee$-gates of $C_{j}$ directly into the $\wedge$-gates at the first level of $C$ that the $j^{\text {th }}$ input of $C$ originally fed into. In the end, we get a circuit $C^{\prime}\left(s^{\prime}\right)$ with $\sqrt{\ell}$ inputs which computes $F^{\prime}\left(s^{\prime}, y_{i}\right)$, where the depth of $C^{\prime}$ is $d+1$ and the number of gates $T_{C^{\prime}}$ in $C^{\prime}$ is at most $T_{C}+r(r-i) \leq T_{C}+r^{2}$.

Corollary 6.8. Let $\mathcal{L}$ be a language in BPP. Suppose for all $x \in$ $\{0,1\}^{n}$ the associated $\mathbf{T M} M(x, y)$ as a function of $y \in\{0,1\}^{r}$ can be expressed as a circuit $C \in \mathcal{C}_{r}^{d}$ for some fixed positive integer $d$, where the number of gates $T_{C}$ in $C$ is at most $r^{c}$ for some fixed positive value $c$. Then, membership of $x$ in $\mathcal{L}$ can be decided by a deterministic computation in time $2^{\log (r)^{\mathcal{O}(1)}}$.

Note that the above corollary applies to derandomize at least partially the randomized approximation algorithm for DNF counting described in Section 6.2. However, the Parity Theorem does not help at all directly in derandomizing the randomized approximation algorithm for GF[2] Polynomial Counting described in Section 6.3, because such a polynomial can easily compute the parity of the number of ones in its input. 
Exercise 6.9. Describe an algorithm that on input positive integers $r, \ell$ and $s$ with $s \leq \ell$, produces $r$ sets $t_{1}, \ldots, t_{r} \subset\{1, \ldots, \ell\}$ such that

- For all $i \in\{1, \ldots, r\},\left|t_{i}\right|=s$.

- For all $i, j \in\{1, \ldots, r\}, j \neq i$.

$$
\left|t_{j} \cap t_{i}\right| \leq \log \left(\frac{\operatorname{rs}\left(1+\frac{s}{\ell}\right)^{s}}{\ell}\right) .
$$

The run time of the algorithm should be polynomial in $r$ and $\ell$. Note that when $s=\sqrt{\ell}$ and $\ell \geq 8$ then $\left|t_{j} \cap t_{i}\right| \leq \log (r)$.

Exercise 6.10. Finish the proof of the above theorem by showing how the distinguishing probability can be converted into prediction probability. 



\section{Acknowledgements}

Avi Wigderson was invited as a Visiting McKay Professor to UC Berkeley for the month of February, 1995. Michael Luby was invited as a Visiting Professor to École Normale Supérieure in Paris for the month of June, 1995. Based on the series of lectures given during these two respective visits, Michael Luby compiled these notes into their current form.

We would like to thank the following people for their help in producing these notes. Preliminary drafts of notes were prepared by Sanjoy Dasgupta, Sean Hallgren, Elizabeth Sweedyk, and Eric Vigoda from UC Berkeley, by Johannes Blömer and Ralph Werchner from the International Computer Science Institute, and by Avy Sharell from Orsey. Eric Vigoda from UC Berkeley and Dana Randall from the Institute for Advanced Studies visited École Normale Supérieure during parts of the month of June, 1995, supported by a grant from the International Branch of the NSF. As part of their activities, Eric helped substantially in compiling these notes during this time, and Dana proofread the entire set of notes, made many good suggestions and found a number of glaring mistakes. 



\section{References}

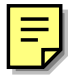

\section{Abbreviations}

- STOC: Proceedings of the ACM Symposium on Theory of Computing

- FOCS: Proceedings of the IEEE Foundations of Computer Science

[1] L. Adleman, "Two theorems on random polynomial time," FOCS, pp. 75-83, 1978.

[2] M. Ajtai, " $\sum_{1}^{1}$-Formulae on finite structures," Annals of Pure and Applied Logic, vol. 24, pp. 1-48, 1983.

[3] M. Ajtai, J. Komlos, and E. Szemeredi, "Deterministic simulation in LOGSPACE," STOC, p. 132, 1987.

[4] W. Alexi, B. Chor, O. Goldreich, and C. Schnorr, "RSA/Rabin functions: Certain parts are as hard as the whole," SIAM J. on Computing, vol. 17, no. 2, pp. 194-209, April 1988.

[5] N. Alon, L. Babai, and A. Itai, "A fast and simple randomized parallel algorithm for the maximal independent set problem," Journal of Algorithms, vol. 7, pp. 567-583, 1986 .

[6] N. Alon and F. R. K. Chung, "Explicit construction of linear sized tolerant networks," Discrete Math, vol. 72, pp. 15-19, 1989.

[7] C. Bennett and J. Gill, "Relative to a random oracle A, $\mathbf{P}^{A} \neq \mathbf{N P}^{A} \neq c o-$ NP $^{A}$ with probability one," Siam J. on Computing, vol. 10, pp. 96-113, 1981.

[8] M. Blum and S. Micali, "How to generate cryptographically strong sequences of pseudo-random bits," SIAM J. on Computing, vol. 13, pp. 850-864, A preliminary version appears in FOCS, 1982, pp. 112-117, 1984. 
[9] J. Cai, "With probability one, a random oracle separates PSPACE from the polynomial-time hierarchy," J. of Computer and System Sci., vol. 38, pp. 68-85, A preliminary version appears in STOC, 1986, pp. 21-29, 1989.

[10] B. Chor and O. Goldreich, "On the power of two-point sampling," Journal of Complexity, vol. 5, pp. 96-106, 1989.

[11] A. Cohen and A. Wigderson, "Dispersers, deterministic amplification, and weak random sources," FOCS, pp. 14-19, 1989.

[12] M. Fredman, J. Komlos, and E. Szemeredi, "Storing a sparse table in O(1) worst case access time," Journal of the ACM, vol. 31, pp. 538-544, 1984.

[13] M. Furst, J. Saxe, and M. Sipser, "Parity, circuits and the polynomial time hierarchy," FOCS, pp. 260-270, 1981.

[14] O. Goldreich, S. Goldwasser, and S. Micali, "How to construct random functions," J. of $A C M$, vol. 33, no. 4, pp. 792-807, A preliminary version appears in FOCS, 1984., 1986.

[15] O. Goldreich and L. Levin, "A hard-core predicate for any one-way function," STOC, pp. 25-32, 1989.

[16] S. Goldwasser and S. Micali, "Probabilistic encryption," J. of Computer and System Sci., vol. 28, pp. 270-299, A preliminary version appears in STOC, 1982, pp. 365-377., 1984.

[17] S. Goldwasser and M. Sipser, "Private coins vs public coins in interactive proof systems," STOC, pp. 59-68, 1986.

[18] J. Håstad, Computational limitations for small depth circuits. Ph.D. thesis, 1986. MIT press.

[19] J. Håstad, R. Impagliazzo, L. A. Levin, and M. Luby, "A pseudo-random generator from any one-way function," SIAM Journal on Computing, vol. 28, no. 4, pp. 1364-1396, 1999.

[20] S. Hoory, N. Linial, and A. Wigderson, "Expander graphs and their applications," Bulletin of the AMS, to appear.

[21] R. Impagliazzo, L. Levin, and M. Luby, "A pseudo-random generator from any one-way function," STOC, pp. 12-24, 1989.

[22] R. Impagliazzo and D. Zuckerman, "How to recycle random bits," FOCS, pp. 248-253, 1990.

[23] R. Karp and M. Luby, "Monte-carlo algorithms for the planar multiterminal network reliability problem," J. of Complexity, vol. 1, pp. 45-64, 1985.

[24] R. Karp, M. Luby, and N. Madras, "Monte-carlo approximation algorithms for enumeration problems," J. of Algorithms, vol. 10, no. 3, pp. 429-448, 1989.

[25] R. Karp, N. Pippenger, and M. Sipser, "Expanders, randomness, or time versus space," First Annual Conference on Structure in Complexity Theory, pp. 325-329, 1986.

[26] M. Karpinski and M. Luby, "Approximating the number of solutions to a GF[2] formula," Journal of Algorithms, vol. 14, no. 2, pp. 280-287, March 1993.

[27] A. Lubotzky, R. Phillips, and P. Sarnak, "Explicit expanders and the ramanujan conjectures," STOC, pp. 240-246, (See also: A. Lubotzky, R. Phillips, P. Sarnak. "Ramanujan graphs," Combinatorica, vol. 8, 1988, pp. 261-277)., 1986. 
[28] M. Luby, "A simple parallel algorithm for the maximal independent set problem," SIAM J. on Computing, vol. 15, no. 4, pp. 1036-1053, November 1986.

[29] G. Margulis, "Explicit group-theoretical constructions of combinatorial schemes and their application to the design of expanders and superconcentrators," Problemy Peredachi Informatsii, vol. 24, pp. 51-60, (in Russian). (English translation in Problems of Information Transmission, vol. 24, 1988, pp. 39-46)., 1988.

[30] N. Nisan, "Pseudorandom bits for constant depth circuits," Combinatorica, vol. 1, pp. 63-70, 1991.

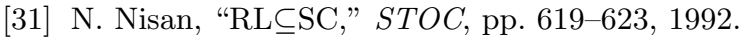

[32] N. Nisan and A. Wigderson, "Hardness vs. randomness," J. of Comp. Sci. and Sys., vol. 49, no. 2, pp. 149-167, 1994.

[33] N. Nisan and D. Zuckerman, "More deterministic simulation in logspace," STOC, pp. 235-244, 1993.

[34] C. H. Papadimitriou, Computational complexity. 1993. Addison Wesley.

[35] A. Renyi, Probability theory. 1970. North-Holland, Amsterdam.

[36] M. Sipser, "A complexity theoretic approach to randomness," STOC, pp. 330-335, 1983.

[37] M. Sipser, Introduction to the theory of computation. PWS Publishing, 1997.

[38] L. Valiant, "The complexity of computing the permanent," Theoretical Computer Science, no. 8, pp. 189-201, 1979.

[39] L. Valiant and V. Vazirani, "NP is as easy as detecting unique solutions," Theoretical Computer Science, vol. 47, pp. 85-93, 1986.

[40] M. Wegman and J. Carter, "New hash functions and their use in authentication and set equality," Journal of Computer and System Sciences, vol. 22, no. 3, pp. 265-279, 1981.

[41] A. Yao, "Theory and applications of trapdoor functions," FOCS, pp. 80-91, 1982.

[42] A. Yao, "Separating the polynomial-time hierarchy by oracles," FOCS, pp. 1-10, 1985. 
